\title{
BILIPSCHITZ AND QUASISYMMETRIC EXTENSION PROPERTIES
}

\author{
JUSSI VÄISÄLÄ
}

\section{Introduction}

Let $X$ and $Y$ be metric spaces with distance denoted by $|a-b|$. A map $f: X \rightarrow Y$ is called $L$-bilipschitz, $L \geqq 1$, if

$$
|x-y| / L \leqq|f(x)-f(y)| \leqq L|x-y|
$$

for all $x, y \in X$. We say that a set $A \subset X$ has the bilipschitz extension property (abbreviated BLEP) in $(X, Y)$ if there is $L_{0}>1$ such that if $1 \leqq L \leqq L_{0}$, then every $L$-bilipschitz $f: A \rightarrow Y$ has an $L_{1}$-bilipschitz extension $g: X \rightarrow Y$, where $L_{1}=$ $L_{1}(L, A, X, Y) \rightarrow 1$ as $L \rightarrow 1$.

Similarly, $A$ has the quasisymmetric extension property (abbreviated QSEP) in $(X, Y)$ if there is $s_{0}>0$ such that if $0 \leqq s \leqq s_{0}$, then every $s$-quasisymmetric $f: A \rightarrow Y$ has an $s_{1}$-quasisymmetric extension $g: X \rightarrow Y$, where $s_{1}=s_{1}(s, A, X, Y) \rightarrow 0$ as $s \rightarrow 0$. The definition of quasisymmetric maps will be recalled in 2.2.

We also say that $A$ has one of these properties in $X$ if $A$ has this property in $(X, X)$. If $A$ has both the BLEP and the QSEP in $(X, Y)$ or in $X$, we say that $A$ has the extension properties in $(X, Y)$ or in $X$, respectively.

In this paper we consider the case where $X$ is the euclidean $n$-space $R^{n}$ and $Y$ is an inner product space. Without loss of generality, we may assume that $Y$ is a linear subspace of the Hilbert space $l_{2}$. The main results are Theorems 5.5 and 6.2. These give sufficient conditions for a set $A \subset R^{n}$ to have the extension properties, the first one in $R^{n}$, the second one in $\left(R^{n}, Y\right)$. Both conditions are somewhat implicit, but we show that the first one applies to all compact DIFF and PL $(n-1)$-manifolds, the second one to all compact convex sets and to all quasisymmetric $n$-cells.

In a joint paper $\left[\mathrm{TV}_{4}\right]$ with Pekka Tukia, we proved that $R^{p}$ and $S^{p}$ have the extension properties in $R^{n}$ for $p \leqq n-1$. In Section 4 we extend these results to the relative case $\left(R^{n}, Y\right)$.

The basic idea of the extension proofs of the present paper is the same as in $\left[\mathrm{TV}_{4}\right]$ : We choose a suitable triangulation of $R^{n} \backslash A$, define the extension $g$ at the vertices, and extend affinely to the simplexes. Thus $g$ will be PL outside $A$. However, 
to define $g$ at the vertices, we must replace the rather explicit constructions of [ $\left.\mathrm{TV}_{4}\right]$ by an auxiliary approximation theorem, which will be given in Section 3.

In Section 7 we give several examples of sets $A \subset R^{n}$ which do not have the extension properties in $R^{n}$ or in $\left(R^{n}, Y\right)$. It is not easy to find an example which has only one of these properties. In fact, I conjecture that if $A$ has the QSEP in $R^{n}$, it has also the BLEP, and that for $n \neq 4$ the proof can be based on the ideas of [ $\left.T V_{5}\right]$ together with careful estimates on the bilipschitz constants. In 7.5 we give an example of a set $A \subset R^{2}$ which has the BLEP but not the QSEP in $R^{2}$. However, I do not know of any such example where $A$ is connected.

I thank Jouni Luukkainen and Pekka Tukia for reading various drafts of this paper and for several valuable remarks and corrections.

\section{Preliminaries}

In this section we give the basic notation and terminology used in this paper, some properties of quasisymmetric maps, and elementary results on affine and PL maps.

2.1. Notation. We let $l_{2}$ denote the Hilbert space of all square summable sequences of real numbers. Let $\left(e_{1}, e_{2}, \ldots\right)$ be its natural basis. We identify the euclidean $n$-space $R^{n}$ with the linear subspace of $l_{2}$ spanned by $e_{1}, \ldots, e_{n}$. Then $R^{p} \subset R^{n}$ for $p \leqq n$. Open balls in $R^{n}$ are written as $B^{n}(x, r)$ and spheres as $S^{n-1}(x, r)$; the superscript may be dropped. We also set

$$
\begin{gathered}
B^{n}(r)=B^{n}(0, r), \quad B^{n}=B^{n}(1), \quad S^{n-1}(r)=S^{n-1}(0, r), \quad S^{n-1}=S^{n-1}(1), \\
R_{+}^{n}=\left\{x \in R^{n}: x_{n} \geqq 0\right\}, \quad B_{+}^{n}=B^{n} \cap R_{+}^{n} .
\end{gathered}
$$

If $A \subset l_{2}$, we let $T(A)$ denote the affine subspace spanned by $A$. In each metric space, $|a-b|$ denotes the distance between $a$ and $b$. If $f$ and $g$ are maps into $l_{2}$, defined on a set $X$, we set

$$
\|f-g\|_{X}=\sup \{|f(x)-g(x)|: x \in X\} .
$$

If $f$ is a bounded linear map between normed spaces, we let $|f|$ denote its sup-norm.

2.2. Quasisymmetric maps. These maps were introduced in $\left[\mathrm{TV}_{1}\right]$. We recall the definition. Let $X$ and $Y$ be metric spaces. An embedding $f: X \rightarrow Y$ is quasisymmetric (abbreviated QS) if there is a homeomorphism $\eta: R_{+}^{1} \rightarrow R_{+}^{1}$ such that if $a, b, x \in X$ with $|a-x| \leqq t|b-x|$, then $|f(a)-f(x)| \leqq \eta(t)|f(b)-f(x)|$. We also say that $f$ is $\eta-\mathrm{QS}$. If $s>0$, we say that $f$ is $s-\mathrm{QS}$ if $f$ is QS and satisfies the following condition: If $t \leqq 1 / s$ and if $a, b, x \in X$ with $|a-x| \leqq t|b-x|$, then $|f(a)-f(x)| \leqq$ $(t+s)|f(b)-f(x)|$. 
In $\left[\mathrm{TV}_{4}\right]$ we used a slightly different definition of $s$-quasisymmetry. We said that $f$ is $s-\mathrm{QS}$ if it is $\eta-\mathrm{QS}$ for some $\eta$ in

$$
N(\mathrm{id}, s)=\{\eta:|\eta(t)-t| \leqq s \text { for } 0 \leqq t \leqq 1 / s\} .
$$

Clearly this condition implies that $f$ is $s-$ QS in the sense given above. Conversely, if $f$ is $s-\mathrm{QS}$, then for every $s^{\prime}>s$ there is $\eta \in N\left(\mathrm{id}, s^{\prime}\right)$ such that $f$ is $\eta-\mathrm{QS}$.

We say that $f$ is a similarity or $0-$ QS if there is $L>0$ such that $|f(x)-f(y)|=$ $L|x-y|$ for all $x, y \in X$. In other words, $f$ is $\eta-$ QS with $\eta=$ id. Every $L$-bilipschitz map is $s-\mathrm{QS}$ with $s=\left(L^{2}-1\right)^{1 / 2}$. If $|f(x)-f(y)|=|x-y|$ for all $x, y \in X, f$ is an isometry. An isometry need not be surjective.

If $G$ is open in $R^{n}, n \geqq 2$, an $\eta-\mathrm{QS}$ map $f: G \rightarrow R^{n}$ is $K$-quasiconformal (abbreviated $K-\mathrm{QC}$ ) with $K=\eta(1)^{n-1}$. The converse is not in general true but a

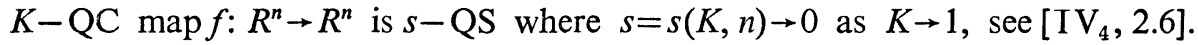

It is often a laborious task to prove that a given embedding $f: X \rightarrow Y$ is QS, since one must consider all triples $a, b, x \in X$. However, it is often possible to exclude triples where the ratio $t=|a-x| /|b-x|$ is small or large. See, for example [IV $2.16,3.10]$. For connected spaces, we prove the following useful result:

2.3. Lemma. Let $X$ and $Y$ be metric spaces with $X$ connected. Suppose that $0<s \leqq 1 / 4$ and that $f: X \rightarrow Y$ is a nonconstant continuous map such that

$$
|f(a)-f(x)| \leqq(t+s)|f(b)-f(x)|
$$

whenever $|a-x|=t|b-x|$ and $1 / 2 \leqq t \leqq 2$. Then $f$ is $\eta-Q S$ with a universal $\eta$, and also $s_{1}-\mathrm{QS}$, where $s_{1}=s_{1}(s) \rightarrow 0$ as $s \rightarrow 0$.

Proof. Let $a, b, x$ be distinct points in $X$ with $|b-x|=r,|a-x|=t r$. Suppose first that $0<t<1 / 2$. We show that (2.4) is also valid in this case. Choose an integer $m \geqq 2$ such that $2^{-m} \leqq t<2^{-m+1}$, and set $t_{0}=t^{1 / m}$. Then $1 / 2 \leqq t_{0}<2^{-1 / 2}$. Since $X$ is connected, we can choose points $b=x_{0}, x_{1}, \ldots, x_{m}=a$ such that $\left|x_{j}-x\right|=t_{0}^{j}$. Since $\left|x_{j+1}-x\right|=t_{0}\left|x_{j}-x\right|$, we have

and hence

$$
\left|f\left(x_{j+1}\right)-f(x)\right| \leqq\left(t_{0}+s\right)\left|f\left(x_{j}\right)-f(x)\right|,
$$

Here

$$
|f(a)-f(x)| \leqq\left(t_{0}+s\right)^{m}|f(b)-f(x)| .
$$

$$
\left(t_{0}+s\right)^{m}-t \leqq\left(2^{-1 / 2}+s\right)^{m}-2^{-1 / 2} \leqq s,
$$

since $2^{-1 / 2}+s \leqq 2^{-1 / 2}+1 / 4<1$. Hence (2.4) is true.

From $\left[\mathrm{TV}_{1}, 2.20\right]$ it follows that $f$ is an embedding. It is easy to verify that $f$ satisfies the conditions of $\left[\mathrm{TV}_{1}, 3.10\right]$ with $\lambda_{1}=\lambda_{2}=3 / 4, h=4 / 3$, and $H=2$. Hence $f$ is $\eta-Q S$ with a universal $\eta$. Indeed, by $\left[\mathrm{TV}_{1}, 3.11\right]$ we can choose $\eta(t)=4 \max \left(t^{5 / 2}, t^{2 / 5}\right)$. 
To show that $f$ is $s_{1}(s)-\mathrm{QS}$ with $s_{1}(s) \rightarrow 0$, let $\varepsilon>0$. Suppose that $t \leqq 1 / \varepsilon$. It suffices to show that there is $\delta=\delta(\varepsilon)>0$ such that if $s \leqq \delta$, then

$$
t^{\prime}=\frac{|f(a)-f(x)|}{|f(b)-f(x)|} \leqq t+\varepsilon .
$$

If $t \leqq 2$, this is true for $s \leqq \varepsilon$. Suppose that $2<t \leqq 1 / \varepsilon$. Choose an integer $n \geqq 2$ such that $2^{n-1}<t \leqq 2^{n}$. Setting $t_{1}=t^{1 / n}$ we have $1<t_{1} \leqq 2$. Since $X$ is connected, we can choose points $b=y_{0}, \ldots, y_{n}=a$ such that $\left|y_{j}-x\right|=t_{1}^{j} r$. Then

which implies

$$
\left|f\left(y_{j+1}\right)-f(x)\right| \leqq\left(t_{1}+s\right)\left|f\left(y_{j}\right)-f(x)\right|,
$$

Thus $t^{\prime} \leqq t+s^{\prime}$ with

$$
|f(a)-f(x)| \leqq\left(t_{1}+s\right)^{n}|f(b)-f(x)| .
$$

$$
s^{\prime}=\left(t_{1}+s\right)^{n}-t_{1}^{n} \leqq(2+s)^{n}-2^{n}=s_{1}(s, n) .
$$

Since $2^{n-1}<t \leqq 1 / \varepsilon, n$ has an upper bound of the form $n \leqq n_{1}(\varepsilon)$. Hence $s^{\prime} \leqq$ $s_{1}\left(s, n_{1}(\varepsilon)\right) \rightarrow 0$ as $s \rightarrow 0$, and thus $s^{\prime} \leqq \varepsilon$ for small $s$.

2.5. Remark. It follows from the proof of 2.3 that for a connected $X, f$ is $s-\mathrm{QS}$ if it satisfies (2.4) for $t \in[1 / 2,1 / s]$ and if $s \leqq 1 / 4$. This is an improvement of $\left[\mathrm{TV}_{4}, 2.4\right]$.

2.6. Simplexes and affine maps. Let $k \geqq 1$ and let $\Delta=a_{0} \ldots a_{k}$ be a $k$-simplex in $l_{2}$ with vertices $a_{0}, \ldots, a_{k}$. We let $b_{j}$ denote the distance of $a_{j}$ from the $(k-1)$-plane spanned by the opposite face, and we set

$$
b(\Delta)=\min \left(b_{0}, \ldots, b_{k}\right) .
$$

The diameter $d(\Delta)$ of $\Delta$ is the largest edge $\left|a_{i}-a_{j}\right|$. The number

$$
\varrho(\Delta)=d(\Delta) / b(\Delta) \geqq 1
$$

is called the flatness of $\Delta$. We let $\Delta^{0}$ denote the set of vertices of $\Delta$.

Let $T \subset l_{2}$ be a finite-dimensional plane (affine subspace), and let $f: T \rightarrow l_{2}$ be affine. We let $L_{f}=L(f)$ and $l_{f}=l(f)$ denote the smallest and the largest number, respectively, such that

$$
l_{f}|x-y| \leqq|f(x)-f(y)| \leqq L_{f}|x-y|
$$

for all $x, y \in T$. Thus $f$ is a similarity if and only if $l_{f}=L_{f}>0$, and an isometry if and only if $l_{f}=L_{f}=1$. Moreover, $f$ is injective if and only if $l_{f}>0$. In this case, the number $H_{f}=L_{f} / l_{f}$ is the metric dilatation of $f$.

Recall that an origin-preserving isometry of an inner product space into an inner product space is linear and preserves the inner product. Such a map is called an orthogonal map. A sense-preserving orthogonal map $R^{n} \rightarrow R^{n}$ is called a rotation.

2.7. Lemma. Suppose that $\Delta \subset l_{2}$ is an n-simplex, that $f: \Delta \rightarrow l_{2}$ is affine and that $h: \Delta \rightarrow l_{2}$ is a similarity such that

$$
|h(v)-f(v)| \leqq \alpha L_{h} b(\Delta) /(n+1)
$$


for every vertex $v$ of $\Delta$, where $0 \leqq \alpha \leqq 1 / 2$. Then

$$
L_{f} \leqq L_{h}(1+2 \alpha), l_{f} \geqq L_{h} /(1+2 \alpha), H_{f} \leqq(1+2 \alpha)^{2} .
$$

If $\Delta \subset R^{n}$ and $f, h: \Delta \rightarrow R^{n}$, then $h$ is sense-preserving if and only if $f$ is sense-preserving.

Proof. Extend $h$ to a bijective similarity $h_{1}: l_{2} \rightarrow l_{2}$. Replacing $f$ by $h_{1}^{-1} f$ we may assume that $h=\mathrm{id}$. The proof of $\left[\mathrm{TV}_{4}, 3.3\right]$ is then valid also in the present situation.

2.8. Lemma. Let $\Delta=a_{0} \ldots a_{k}$ be a $k$-simplex in $R^{n}$ with $a_{0}=0$. Suppose that $g: T(\Delta) \rightarrow R^{n}$ is an orthogonal map such that $g a_{j}=a_{j}$ for $0 \leqq j \leqq k-1$ and $\left|g a_{k}-a_{k}\right| \leqq$ $\leqq \delta$. Then there is an orthogonal map $u: R^{n} \rightarrow R^{n}$ such that $u g \mid \Delta=\mathrm{id}$ and $|u-\mathrm{id}| \leqq$ $\delta / b(\Delta)$. If $k<n, u$ can be chosen to be a rotation.

Proof. If $k=n$, either $g=\mathrm{id}$ or $g$ is the reflection in $T\left(a_{0}, \ldots, a_{k-1}\right)$. In the first case we choose $u=\mathrm{id}$. In the second case we have $\delta \geqq\left|g a_{k}-a_{k}\right|=2 b_{k} \geqq 2 b(\Delta)$. Since $|g-\mathrm{id}|=2 \leqq \delta / b(\Delta)$, we can choose $u=g$.

Suppose that $k<n$. Let $E$ be the linear subspace of $R^{n}$ spanned by $a_{1}, \ldots, a_{k-1}$. Let $q_{1}: R^{n} \rightarrow E$ and $q_{2}: R^{n} \rightarrow E^{\perp}$ be the orthogonal projections. Let $T$ be a twodimensional linear subspace of $E^{\perp}$ containing the vectors $x_{1}=q_{2} g a_{k}$ and $x_{2}=q_{2} a_{k}$. Since $g a_{k}=g q_{1} a_{k}+g q_{2} a_{k}$ and since $g \mid E=\mathrm{id}$, we have $g x_{2}=x_{1}$, and thus $\left|x_{1}\right|=\left|x_{2}\right|$. Consequently, there is a rotation $u$ of $T$ with $u x_{1}=x_{2}$. Extend $u$ to a rotation $u: R^{n} \rightarrow$ $R^{n}$ with $u \mid T^{\perp}=\mathrm{id}$. Since $\left|x_{1}-x_{2}\right| \leqq \delta,|u-\mathrm{id}| \leqq \delta /\left|x_{2}\right|$. Here $\left|x_{2}\right|=d\left(a_{k}, E\right) \geqq b(\Delta)$, and the lemma follows.

2.9. Lemma. Let $\Delta=a_{0} \ldots a_{p}$ be a p-simplex in $R^{n}$ with $a_{0}=0$. Suppose that $h: T(\Delta) \rightarrow R^{n}$ is an orthogonal map such that $\left|h a_{j}-a_{j}\right| \leqq \delta$ for all $j$. Then there is an orthogonal map $u: R^{n} \rightarrow R^{n}$ such that

$$
u h|\Delta=\mathrm{id},| h-\mathrm{id}|\leqq| u-\mathrm{id} \mid \leqq b(\Delta)^{-1} p(1+\varrho(\Delta))^{p-1} \delta .
$$

If $p<n, u$ can be chosen to be a rotation.

Proof. We define inductively orthogonal maps $u_{k}: R^{n} \rightarrow R^{n}, 0 \leqq k \leqq p$, as follows: Let $u_{0}=\mathrm{id}$. Assume that we have constructed $u_{0}, \ldots, u_{k-1}$ such that setting $g_{j}=$ $u_{j-1} \ldots u_{0}$, we have $g_{j} h a_{i}=a_{i}$ for $i<j \leqq k$. Apply 2.8 with the substitution

$k \mapsto k, \Delta \mapsto \Delta_{k}=a_{0} \ldots a_{k}, g \mapsto g_{k} h \mid T\left(\Delta_{k}\right), \delta \mapsto \delta_{k}=\max \left\{\left|g_{k} h a_{i}-a_{i}\right|: 1 \leqq i \leqq p\right\}$.

We obtain an orthogonal map $u_{k}: R^{n} \rightarrow R^{n}$ such that $u_{k} g_{k} h \mid \Delta_{k}=\mathrm{id}$ and $\left|u_{k}-\mathrm{id}\right| \leqq$ $\delta_{k} / b\left(\Delta_{k}\right)$. Thus

$$
g_{k+1} h\left|\Delta_{k}=\mathrm{id},\right| u_{k}-\mathrm{id} \mid \leqq b(\Delta)^{-1} \delta_{k} .
$$

We show by induction that

$$
\delta_{k} \leqq(1+\varrho)^{k-1} \delta,
$$

where $\varrho=\varrho(\Delta)$. This is clearly true for $k=1$. Suppose that $(2.10)$ holds for $k<s$. 
Since $\left|a_{i}\right| \leqq d(\Delta) \leqq \varrho b(\Delta)$, we obtain

$$
\begin{aligned}
\left|g_{s} h a_{i}-a_{i}\right| & \leqq\left|u_{s-1} g_{s-1} h a_{i}-g_{s-1} h a_{i}\right|+\left|g_{s-1} h a_{i}-a_{i}\right| \\
& \leqq b(\Delta)^{-1} \delta_{s-1}\left|a_{i}\right|+\delta_{s-1} \\
& \leqq(\varrho+1) \delta_{s-1} \leqq(\varrho+1)^{s-1} \delta,
\end{aligned}
$$

which gives (2.10) for $k=s$.

Since

and

$$
\left|g_{k+1}-\mathrm{id}\right| \leqq\left|u_{k} g_{k}-g_{k}\right|+\left|g_{k}-\mathrm{id}\right| \leqq\left|u_{k}-\mathrm{id}\right|+\left|g_{k}-\mathrm{id}\right|
$$

we obtain

$$
\left|u_{k}-\mathrm{id}\right| \leqq b(\Delta)^{-1} \delta_{k} \leqq b(\Delta)^{-1}(1+\varrho)^{p-1} \delta,
$$

$$
\left|g_{p+1}-\mathrm{id}\right| \leqq\left|u_{1}-\mathrm{id}\right|+\ldots+\left|u_{p}-\mathrm{id}\right| \leqq b(\Delta)^{-1} p(1+\varrho)^{p-1} \delta .
$$

Since $h=g_{p+1}^{-1} \mid T(\Delta)$, the lemma is true with $u=g_{p+1}$.

2.11. Lemma. Let $\Delta \subset l_{2}$ be a p-simplex. Suppose that $h, k: T(\Delta) \rightarrow l_{2}$ are similarities such that $|h(z)-k(z)| \leqq \delta$ for all $z \in \Delta^{0}$. Then

$$
\begin{gathered}
\left|L_{h}-L_{k}\right| \leqq 2 \delta / d(\Delta), \\
|h(x)-k(x)| \leqq \delta\left(1+d(\Delta)^{-1} M|x-v|\right)
\end{gathered}
$$

for all $x \in T(\Delta)$ and $v \in \Delta^{0}$, where

$$
M=4+6 \varrho(\Delta) p(1+\varrho(\Delta))^{p-1} .
$$

Proof. Observing that

$$
L_{h} d(\Delta)=d(h \Delta) \leqq d(k \Delta)+2 \delta=L_{k} d(\Delta)+2 \delta
$$

and interchanging the roles of $h$ and $k$, we obtain the first inequality.

To prove the second inequality, we may assume that $\Delta \subset R^{p}$, that $v=0$ and that $h, k: R^{p} \rightarrow R^{n}, n=2 p+1$. Assume first that $h(0)=0=k(0)$. Extend $h$ to a similarity $h_{1}: R^{n} \rightarrow R^{n}$. Then the map $g=\left(L_{h} / L_{k}\right) h_{1}^{-1} k: R^{p} \rightarrow R^{n}$ is an orthogonal map. If $z \in \Delta^{0}$, we have

$$
\begin{aligned}
|g z-z| & \leqq\left|\left(L_{h} / L_{k}\right) h_{1}^{-1} k z-h_{1}^{-1} k z\right|+\left|h_{1}^{-1} k z-z\right| \\
& \leqq\left|L_{h} / L_{k}-1\right| L_{k}|z| / L_{h}+|k z-h z| / L_{h} \leqq 3 \delta / L_{h} .
\end{aligned}
$$

Hence 2.9 implies that

$$
|g-\mathrm{id}| \leqq b(\Delta)^{-1} L_{h}^{-1} M_{1} \delta
$$

with $M_{1}=3 p(1+\varrho(\Delta))^{p-1}$. Consequently

$$
\begin{aligned}
|h-k| & \leqq L_{h}\left|h_{1}^{-1} k-\mathrm{id}\right| \\
& \leqq L_{h}\left|h_{1}^{-1} k-g\right|+L_{h}|g-\mathrm{id}| \\
& \leqq\left|L_{h}-L_{k}\right|+b(\Delta)^{-1} M_{1} \delta \\
& \leqq d(\Delta)^{-1} M \delta / 2 .
\end{aligned}
$$


In the general case, set $h^{\prime}(x)=h(x)-h(0), k^{\prime}(x)=k(x)-k(0)$, and apply the inequality above to the linear maps $h^{\prime}, k^{\prime}$ with $\delta$ replaced by $2 \delta$. We obtain

$$
|h(x)-k(x)| \leqq|h(0)-k(0)|+\left|h^{\prime}(x)-k^{\prime}(x)\right| \leqq \delta\left(1+d(\Delta)^{-1} M|x|\right) .
$$

2.12. Lemma. Let $\Delta \subset R^{n}$ be an (n-1)-simplex. Suppose that $h, k: R^{n} \rightarrow R^{n}$ are sense-preserving similarities such that $|h(z)-k(z)| \leqq \delta$ for all $z \in \Delta^{0}$. Then the inequalities of 2.11 are true with $p=n-1$ for all $x \in R^{n}$ and $v \in \Delta^{0}$.

Proof. We repeat the proof of 2.11 with a slight modification. When applying 2.9 we first obtain a rotation $u: R^{n} \rightarrow R^{n}$ satisfying the inequality of 2.9. Since $u g$ is a rotation with $u g \mid \Delta=\mathrm{id}$, we have $u=g^{-1}$, which implies $|u-\mathrm{id}|=|g-\mathrm{id}|$. The rest of the proof is unchanged.

2.13. Suppose that $K$ is a simplicial complex. We say that a map $f:|K| \rightarrow l_{2}$ is simplicial if $f$ is affine on every simplex of $K$. We let $K^{0}$ denote the set of vertices of $K$.

The proof of the following lemma is based on an idea of $\mathbf{J}$. Luukkainen.

2.14. Lemma. Let $K$ be a finite simplicial complex in $l_{2}$. Then there is $\alpha_{0}=\alpha_{0}(K)>$ 0 such that if $0 \leqq \alpha \leqq \alpha_{0}, f:|K| \rightarrow l_{2}$ is simplicial, $h: K^{0} \rightarrow l_{2}$ is a similarity and $\|f-h\|_{K^{0}} \leqq \alpha L_{h}$, then

$$
L_{h}|x-y| / \Lambda \leqq|f(x)-f(y)| \leqq \Lambda L_{h}|x-y|
$$

for all $x, y \in|K|$, where $\Lambda=\Lambda(\alpha, K) \rightarrow 1$ as $\alpha \rightarrow 0$.

If $u:|K| \rightarrow l_{2}$ is a similarity, one can choose $\alpha_{0}(u K)=L_{u} \alpha_{0}(K)$ and $\Lambda(\alpha, u K)=$ $\Lambda\left(\alpha / L_{u}, K\right)$.

Proof. The last statement of the lemma is clear. Replacing $f$ and $h$ by $f / L_{h}$ and $h / L_{h}$, we may assume that $h$ is an isometry.

We say that a pair $\Delta_{1}, \Delta_{2}$ of simplexes is a proper simplex pair if $\Delta_{1} \varangle \Delta_{2}$ and $\Delta_{2} \leftarrow \Delta_{1}$. If $K$ has no proper simplex pairs, the lemma follows from 2.7. The lemma is clearly true if $\operatorname{dim} K=0$. Let $0 \leqq p \leqq q \geqq 1$ be integers. We make the inductive hypothesis that the lemma holds for all $K$ such that if $\left(\Delta_{1}, \Delta_{2}\right)$ is a proper simplex pair of $K$ with $\operatorname{dim} \Delta_{1} \leqq \operatorname{dim} \Delta_{2}$, then either $\operatorname{dim} \Delta_{2}<q$ or $\operatorname{dim} \Delta_{2}=q$ and $\operatorname{dim} \Delta_{1}<p$. It suffices to prove the lemma in the case where $K$ has exactly two principal simplexes $\Delta_{1}, \Delta_{2}$ with $\operatorname{dim} \Delta_{1}=p, \operatorname{dim} \Delta_{2}=q$. Extending $h$ to a bijective isometry $h_{1}$ of $l_{2}$ and replacing $f$ by $h_{1}^{-1} f$, we may assume that $h=$ id. Since $f$-id is simplicial, we have $\|f-\mathrm{id}\|_{|K|} \leqq \alpha$.

Set $\Delta=\Delta_{1} \cap \Delta_{2}$. Let $x \in \Delta_{1} \backslash \Delta, y \in \Delta_{2} \backslash \Delta$. We must find an upper and a lower bound for $|f(x)-f(y)| /|x-y|$.

Case 1. $\Delta=\emptyset$. Now $d\left(\Delta_{1}, \Delta_{2}\right)=\delta>0$, and

$$
\begin{aligned}
& |f(x)-f(y)| \leqq|x-y|+2 \alpha \leqq(1+2 \alpha / \delta)|x-y|, \\
& |f(x)-f(y)| \geqq|x-y|-2 \alpha \geqq(1-2 \alpha / \delta)|x-y| .
\end{aligned}
$$


Hence we can choose

$$
\begin{aligned}
& \alpha_{0}(K)=\min \left(\delta / 3, \alpha_{0}\left(\Delta_{1}\right), \alpha_{0}\left(\Delta_{2}\right), \Lambda(\alpha, K)\right) \\
& =\max \left((1-2 \alpha / \delta)^{-1}, \Lambda\left(\alpha, \Delta_{1}\right), \Lambda\left(\alpha, \Delta_{2}\right)\right) .
\end{aligned}
$$

Case 2. $\Delta \neq \emptyset$. By the inductive hypothesis, the lemma holds for the complexes $K \backslash\left\{\Delta_{1}\right\}$ and $K \backslash\left\{\Delta_{2}\right\}$. Choose $\alpha_{0}>0$ and a function $\Lambda:\left[0, \alpha_{0}\right] \rightarrow[1, \infty)$ with the properties given by the lemma for these complexes. Let $0 \leqq \alpha \leqq \alpha_{0}$. Choose $a \in \Delta$. Then $x \in a b$ and $y \in a c$ for some $b \in \partial \Delta_{1} \backslash \Delta$ and $c \in \partial \Delta_{2} \backslash \Delta$. We may assume that

$$
\frac{|b-a|}{|x-a|} \geqq \frac{|c-a|}{|y-a|}=\lambda \text {. }
$$

Then there is $z \in x b$ such that $|z-a|=\lambda|x-a|$, and thus $z-c=\lambda(x-y)$. Since $f$ is affine on $a b$ and on $a c$, we have $f(z)-f(c)=\lambda(f(x)-f(y))$, and hence

$$
|f(x)-f(y)|=\lambda^{-1}|f(z)-f(c)| \leqq \Lambda \lambda^{-1}|z-c|=\Lambda|x-y|,
$$

and similarly

$$
|f(x)-f(y)| \geqq \Lambda^{-1}|x-y| .
$$

Hence one can choose $\alpha_{0}(K)=\alpha_{0}$ and $\Lambda(\alpha, K)=\Lambda(\alpha)$.

We next give an estimate for the flatness of a simplex. This will be needed in the proof of 5.19 .

2.15. Lemma. Suppose that $\Delta \subset R^{p}$ is a p-simplex with $\varrho(\Delta) \leqq M$. Suppose that $v$ is a point in $R^{p+1}$ such that $v_{p+1} \geqq \delta d(\Delta), \delta>0$, and $d(v, \Delta) \leqq c d(\Delta)$. Then $v \Delta$ is $a(p+1)$-simplex with $\varrho(v \Delta) \leqq M_{1}(M, \delta, c, p)$.

Proof. Let $a_{0}, \ldots, a_{p}$ be the vertices of $\Delta$, and set $\Delta_{1}=v \Delta$. We first derive a lower bound for the numbers $b_{j}=b_{j}\left(\Delta_{1}\right)$ (see 2.6). Clearly $b_{p+1}=v_{p+1} \geqq \delta d(\Delta)$. For $0 \leqq j \leqq p$ we may assume $j=0$. Writing $\Delta_{0}=a_{1} \ldots a_{p} v$ we have $b_{0}=(p+1) m_{p+1}\left(\Delta_{1}\right) /$ $m_{p}\left(\Delta_{0}\right)$, where $m_{p}$ is the $p$-measure. For any $p$-simplex $\sigma$ we have

$$
p ! m_{p}(\sigma) \geqq b(\sigma)^{p-1} d(\sigma),
$$

which can easily be proved by induction on $p$. This implies

Since

$$
m_{p+1}\left(\Delta_{1}\right) \geqq \frac{m_{p}(\Delta) \delta d(\Delta)}{p+1} \geqq \frac{\delta b(\Delta)^{p-1} d(\Delta)^{2}}{(p+1) p !} .
$$

we obtain

$$
m_{p}\left(\Delta_{0}\right) \leqq d\left(\Delta_{0}\right)^{p} \leqq(1+c)^{p} d(\Delta)^{p},
$$

$$
b_{0} \geqq \frac{\delta d(\Delta)}{p !(1+c)^{p} M^{p-1}} .
$$

Since $d\left(\Delta_{1}\right) \leqq(1+c) d(\Delta)$, the lemma is true with

$$
M_{1}=p !(1+c)^{p+1} M^{p-1} / \delta
$$




\section{Approximation by similarities and by isometries}

Intuitively, an $L$-bilipschitz map with small $L$ is close to an isometry, and an $s-$ QS map with small $s$ is close to a similarity. We shall give a precise meaning for this in this section.

3.1. Theorem. Let $A \subset R^{p}$ be compact, let $Y$ be a linear subspace of $l_{2}$ with $\operatorname{dim} Y \geqq p$, and let $f: A \rightarrow Y$ be $s-\mathrm{QS}$. Then there is a similarity $h: R^{p} \rightarrow Y$ such that

$$
\|h-f\|_{A} \leqq \chi(s, p) L_{l h} d(A),
$$

where $s \mapsto \chi(s, p)$ is an increasing function and $\chi(s, p) \rightarrow 0$ as $s \rightarrow 0$.

Iff is $L$-bilipschitz and $s=\left(L^{2}-1\right)^{1 / 2}$, then $h$ can be chosen to be an isometry.

Proof. Suppose that the first part of the theorem is false. Then there exist $\lambda>0$ and a sequence $f_{j}: A_{j} \rightarrow Y_{j}$ of $\eta_{j}-\mathrm{QS}$ maps such that each $A_{j}$ is compact in $R^{p}, \eta_{j} \in N(\mathrm{id}, 1 / j)$, and

$$
\left\|f_{j}-h\right\|_{A_{j}} \geqq \lambda L_{h} d\left(A_{j}\right)
$$

for every similarity $h: R^{p} \rightarrow Y_{j}$. Passing to a subsequence we may assume that $\operatorname{dim} T\left(A_{j}\right)=k$ does not depend on $j$. For each positive integer $j$ we choose points $a_{j}^{0}, \ldots, a_{j}^{k} \in A_{j}$ as follows: Let $a_{j}^{0} \in A_{j}$ be arbitrary, and let $a_{j}^{i+1}$ be a point $x \in A_{j}$ at which the distance $d\left(x, T\left(a_{j}^{0}, \ldots, a_{j}^{i}\right)\right)$ is maximal.

Using auxiliary similarities of $R^{p}$ and $l_{2}$, we may assume that $R^{p} \subset Y_{j}$ and that

$$
\begin{gathered}
a_{j}^{0}=0, \quad a_{j}^{1}=e_{1}, \quad a_{j}^{i} \in \operatorname{int} R_{+}^{i} \text { for } 2 \leqq i \leqq k, \\
f\left(a_{j}^{0}\right)=0, \quad f_{j}\left(a_{j}^{1}\right)=e_{1}, \quad f_{j}\left(a_{j}^{i}\right) \in R_{+}^{i} \text { for } 2 \leqq i \leqq k .
\end{gathered}
$$

Then $A_{j} \subset \bar{B}^{k}$ and $1 \leqq d\left(A_{j}\right) \leqq 2$. Applying $\left[\mathrm{TV}_{1}, 2.5\right]$ with the substitution $A \mapsto$ $\left\{0, e_{1}\right\}, B \mapsto A_{j}, f \mapsto f_{j}$, yields

$$
d\left(f_{j} A_{j}\right) \leqq 2 \eta_{j}\left(d\left(A_{j}\right)\right) \leqq 2 \eta_{j}(2) \leqq 2(2+1 / j) \leqq 5
$$

for $j \geqq 2$. Applying (3.3) with $h=$ id we find $x_{j} \in A_{j}$ such that

$$
\left|f_{j}\left(x_{j}\right)-x_{j}\right| \geqq \lambda
$$

for all $j \geqq 2$. Passing to a subsequence and performing an auxiliary isometry $\varphi$ of $l_{2}$ with $\varphi \mid R^{k}=\mathrm{id}$, we may assume that the following sequences converge as $j \rightarrow \infty$ :

$$
\begin{gathered}
a_{j}^{i} \rightarrow a^{i} \in \bar{B}_{+}^{i}, \quad 0 \leqq i \leqq k, \\
f_{j}\left(a_{j}^{i}\right) \rightarrow b^{i} \in \bar{B}_{+}^{i}(5), \quad 0 \leqq i \leqq k, \\
x_{j} \rightarrow x_{0} \in \bar{B}^{k}, \\
f_{j}\left(x_{j}\right) \rightarrow y_{0} \in \bar{B}^{k+1}(5) .
\end{gathered}
$$

Moreover, $a^{0}=b^{0}=0$ and $a^{1}=b^{1}=e_{1}$. 
Put $T=T\left(a^{0}, \ldots, a^{k}\right), s=\operatorname{dim} T$. Then $s \leqq k$ and $a^{i} \in \operatorname{int} R_{+}^{i}$ for $i \leqq s$. Since $\eta_{j} \in N(\mathrm{id}, 1 / j)$, we have

$$
\left|f_{j}\left(a_{j}^{2}\right)\right|=\frac{\left|f_{j}\left(a_{j}^{2}\right)-f_{j}(0)\right|}{\left|f_{j}\left(e_{1}\right)-f_{j}(0)\right|} \rightarrow \frac{\left|a^{2}-0\right|}{\left|e_{1}-0\right|}=\left|a^{2}\right| .
$$

Hence $\left|b^{2}\right|=\left|a^{2}\right|$. Changing the roles of 0 and $e_{1}$, a similar argument shows that $\left|b^{2}-e_{1}\right|=\left|a^{2}-e_{1}\right|$. Since $a^{2}, b^{2} \in R_{+}^{2}$, we obtain $a^{2}=b^{2}$. Proceeding inductively, we similarly obtain $a^{i}=b^{i}$ for $0 \leqq i \leqq s$. Since

$$
\lim _{j \rightarrow \infty} \max _{x \in A_{j}} d(x, T)=0
$$

we have $x_{0} \in T$. If $i$ and $l$ are distinct integers on $[0, s]$,

$$
\frac{\left|y_{0}-a^{i}\right|}{\left|a^{l}-a^{i}\right|}=\lim _{j \rightarrow \infty} \frac{\left|f_{j}\left(x_{j}\right)-f_{j}\left(a_{j}^{i}\right)\right|}{\left|f_{j}\left(a_{j}^{l}\right)-f_{j}\left(a_{j}^{i}\right)\right|}=\lim _{j \rightarrow \infty} \frac{\left|x_{j}-a_{j}^{i}\right|}{\left|a_{j}^{l}-a_{j}^{i}\right|}=\frac{\left|x_{0}-a^{i}\right|}{\left|a^{l}-a^{i}\right|} .
$$

Thus $\left|y_{0}-a^{i}\right|=\left|x_{0}-a^{i}\right|$ for $0 \leqq i \leqq s$. Since $a^{0}, \ldots, a^{s}$ are affinely independent in $T$ and $x_{0} \in T$, this implies $x_{0}=y_{0}$. Since $\left|x_{0}-y_{0}\right| \geqq \lambda$, this is a contradiction.

The bilipschitz case could be proved in a similar manner, but it also follows from the QS case. Assume that $f: A \rightarrow Y$ is $L$-bilipschitz. Then $f$ is $s-\mathrm{QS}$ with $s=$ $\left(L^{2}-1\right)^{1 / 2}$. Choose a similarity $h: R^{p} \rightarrow Y$ satisfying (3.2). We may assume that $0 \in A$ and that $h(0)=0$. Then $h_{1}=h / L_{h}$ is an isometry. For each $x \in A$ we have

where

$$
\begin{aligned}
\left|f(x)-h_{1}(x)\right| & \leqq|f(x)-h(x)|+\left|h(x)-h_{1}(x)\right| \\
& \leqq \varkappa(s, p) L_{h} d(A)+\left|1-1 / L_{h}\right||h(x)| \\
& \leqq \chi_{1}(s, p) d(A),
\end{aligned}
$$

$$
\varkappa_{1}(s, p)=L_{h} \varkappa(s, p)+\left|1-L_{h}\right|
$$

On the other hand,

$$
L_{h} d(A)=d(h A) \leqq d(f A)+2 \varkappa(s, p) L_{h} d(A) .
$$

This implies

$$
L_{h} \leqq \frac{L}{1-2 \chi(s, p)}
$$

as soon as $L-1$ is so small that $2 \varkappa(s, p)<1$. Similarly, we obtain a lower bound for $L_{h}$, and (3.4) yields

as $L \rightarrow 1$.

$$
\varkappa_{1}(s, p)=\delta(L, p) \rightarrow 0
$$

3.6. Remarks. 1. Theorem 3.1 is true with (3.2) replaced by the inequality

$$
\|f-h\|_{\boldsymbol{A}} \leqq \varkappa(s, p) d(f A),
$$

replacing $\varkappa$ by another function with the same properties. This follows easily from (3.5). 
2. In the QS case of (3.2) and (3.7) one can always choose $\varkappa(s, p) \leqq 2$. By auxiliary similarities we can normalize the situation so that $0 \in A, f(0)=0$, and $d(A)=d(f A)=1$. Then

$$
\|f-\mathrm{id}\|_{\boldsymbol{A}} \leqq d(f A)+d(A) \leqq 2 .
$$

This observation is due to J. Luukkainen.

We next prove converse results of 3.1. These are not needed in the rest of the paper.

3.8. Theorem. Let $0<\delta<1 / 2$, let $X \subset R^{p}$, and let $f: X \rightarrow l_{2}$ be a map such that for every bounded $A \subset X$ there is an isometry $h: R^{p} \rightarrow l_{2}$ such that $\|h-f\|_{A} \leqq$ $\delta d(A)$. Then $f$ is L-bilipschitz with $L=(1-2 \delta)^{-1}$.

Proof. Let $a, b \in X$ with $a \neq b$. Set $A=\{a, b\}$, and choose the corresponding isometry $h$. Now

and similarly

$$
\begin{gathered}
|f(a)-f(b)| \leqq|h(a)-h(b)|+|h(a)-f(a)|+|h(b)-f(b)| \\
\leqq(1+2 \delta)|a-b| \leqq(1-2 \delta)^{-1}|a-b|,
\end{gathered}
$$

$$
|f(a)-f(b)| \geqq(1-2 \delta)|a-b| \text {. }
$$

3.9. Theorem. Let $0<x \leqq 1 / 25$, let $X \subset R^{p}$ be connected, and let $f: X \rightarrow l_{2}$ be a map such that for every bounded $A \subset X$ there is a similarity $h: R^{p} \rightarrow l_{2}$ such that $\|h-f\|_{A} \leqq \varkappa L_{h} d(A)$. Then fis $s-\mathrm{QS}$, where $s=s(\chi) \rightarrow 0$ as $\chi \rightarrow 0$.

Proof. We first show that $f$ is injective. Let $a, b \in X$ with $a \neq b$. Set $A=\{a, b\}$ and choose the corresponding similarity $h$. Then

$$
|f(a)-f(b)| \geqq(1-2 x)|h(a)-h(b)|>0 .
$$

Now assume that $a, b, x$ are distinct points in $X$ with $|a-x|=t|b-x|$. Set $A=$ $\{a, b, x\}$ and choose the corresponding similarity $h: R^{p} \rightarrow l_{2}$. Since

$$
|f(b)-f(x)| \geqq|h(b)-h(x)|-2 x L_{h} d(A)=L_{h}|b-x|-2 x L_{h} d(A),
$$

we obtain

Since

$$
\begin{gathered}
|f(a)-f(x)| \leqq|h(a)-h(x)|+2 \varkappa L_{h} d(A) \\
\leqq t|f(b)-f(x)|+2(1+t) \chi L_{h} d(A) .
\end{gathered}
$$

we obtain

$$
\begin{gathered}
d(A) \leqq|a-x|+|b-x|=(1+t)|b-x|, \\
L_{h} d(A) \leqq(1+t)|h(b)-h(x)| \\
\leqq(1+t)|f(b)-f(x)|+2(1+t) x L_{h} d(A) .
\end{gathered}
$$

Assume $t \leqq x^{-1 / 2}$. Since $x \leqq 1 / 25$, we have $2(1+t) x<1 / 2$, and thus

$$
L_{h} d(A) \leqq 2(1+t)|f(b)-f(x)| .
$$


Consequently, $|f(a)-f(x)|=t^{\prime}|f(b)-f(x)|$ with

$$
t^{\prime} \leqq t+4 \chi(1+t)^{2} \text {. }
$$

Assuming $t \leqq \varkappa^{-1 / 4}$ this implies

$$
t^{\prime} \leqq t+9 x^{1 / 2} .
$$

Hence, if $f$ is QS, it is $s-\mathrm{QS}$ with

$$
s=s(\varkappa)=\max \left(\varkappa^{1 / 4}, 9 x^{1 / 2}\right) .
$$

To show that $f$ is QS, we verify that $f$ satisfies the conditions (1) and (2) of [TV $\left.{ }_{1}, 3.10\right]$ with $\lambda_{1}=\lambda_{2}=1 / 2, h=2, H=4$. Since $X$ is connected, it is $\lambda-\mathrm{HD}$. If $t \leqq 2$, then $t \leqq x^{-1 / 4}$, and hence $t^{\prime} \leqq t+9 x^{1 / 2}<4$. If $t \leqq 1 / 4$, then (3.10) implies $t^{\prime} \leqq 1 / 2$. The quasisymmetry of $f$ follows then from the proof of $\left[\mathrm{TV}_{1}, 3.10\right]$ and from $\left[\mathrm{TV}_{1}, 2.21\right]$.

\section{Planes and spheres}

In $\left[\mathrm{TV}_{4}\right]$ we proved that $R^{p}$ and $S^{p}$ have the extension properties in $R^{n}$ for $p<n$. In this section we show that $R^{n}$ can be replaced by $\left(R^{n}, Y\right)$ where $Y$ is any linear subspace of $l_{2}$ with $\operatorname{dim} Y \geqq n$. The result will be needed in Section 6 .

4.1. Theorem. Let $Y$ be a linear subspace of $l_{2}$ with $\operatorname{dim} Y \geqq n$, and let $1 \leqq p \leqq$ $n-1$. Then $R^{p}$ has the extension properties in $\left(R^{n}, Y\right)$. The numbers in the definition of the extension properties do not depend on $Y$, thus $L_{0}=L_{0}(n), L_{1}=L_{1}(L, n), s_{0}=$ $s_{0}(n), s_{1}=s_{1}(s, n)$.

Proof. The proof can be carried out by rewriting the proof of $\left[\mathrm{TV}_{4}, 5.3,5.4\right]$ in this more general setting. However, some modifications have to be made. We shall only give these modifications.

The lemmas of $\left[\mathrm{TV}_{4}\right.$, Section 3] are easily generalized to the new setting and partly given in Section 2 of the present paper. The results of $\left[\mathrm{TV}_{4}\right.$, Section 4] concerning frames are still valid in the general case but in the proofs one cannot make use of the compactness of the space $V_{n}^{0}(Y)$ of all orthonormal $n$-frames of $Y$. However, the uniform differentiability formulas $\left[\mathrm{TV}_{4},(4.2),(4.3)\right]$ of the Gram-Schmidt map $G: V_{n}(Y) \rightarrow V_{n}^{0}(Y)$ are still valid in some neighborhood $N$ of $V_{n}^{0}(Y)$ in $V_{n}(Y)$, as easily follows from the definition of $G$. Hence we obtain the interpolation lemma $\left[\mathrm{TV}_{4}, 4.4\right]$ with $R^{n}$ replaced by $Y$. The crucial extension lemma $\left[\mathrm{TV}_{4}, 4.9\right]$ also remains valid with $R^{n}$ replaced by $Y$. Although $V_{n}^{0}(Y)$ is not necessarily compact, $G$ is still uniformly continuous in a neighborhood of it. On the other hand, we cannot use the diagonal process to conclude that it suffices to define the map $u: \mathscr{I}(p) \rightarrow V_{n}^{0}(Y)$ only on $\mathscr{I}(p, k)$. Instead, we give a direct construction of $u$ on the whole $\mathscr{I}(p)$.

We again start with the cube $Q_{0}=J^{p}$ and define $u_{Q_{0}}=u_{0}$. Next we inductively define $u_{Q_{j}}$ for $Q_{j}=2^{j} J^{p}$ directly by $u_{Q_{j-1}}$ for all positive integers $j$. For each $j$ we consider the family of the $3^{p}-1$ cubes $R \in \mathscr{I}_{j}(p)$ with $R \sim Q_{j}, R \neq Q_{j}$, and define 
$u_{R}$ directly by $u_{Q_{j}}$. Next we define $u_{P_{R}}$ for the principal subcubes $P_{R}$ of these cubes $R$ directly by $u_{R}$. Then we apply the generalized version of [ $\left[\mathrm{V}_{4}, 4.4\right]$ to define $u_{Q}$ for every $Q \in \mathscr{I}_{j-1}(p)$ in the convex hull $E_{j-1}$ of the union of these principal subcubes, except for those $Q$ for which $u_{Q}$ has already been defined directly by $u_{Q_{j-1}}$. Proceeding in this manner, it is easy to see that we obtain a map $u: \mathscr{I}(p) \rightarrow V_{n}^{0}(Y)$ with the desired properties provided that $q \leqq 2^{-p-4}$.

The extension $g: R^{n} \rightarrow Y$ of the given $L$-bilipschitz or $s-\mathrm{QS}$ map $f: R^{p} \rightarrow Y$ can now be constructed and its continuity proved as in the proofs of Theorems 5.3 and 5.4 of $\left[\mathrm{TV}_{4}\right]$. However, we must give a new proof for the fact that $g$ is $L_{1}$-bilipschitz or $s_{1}-\mathrm{QS}$, because the old one was based on the convexity of $g R^{n}$ in the bilipschitz case and on the theory of QC maps in the QS case. We shall prove the QS case. The proof for the bilipschitz case is similar but easier; observe that the convexity of $R^{p}$ implies that $g$ is lipschitz.

To show that $g$ is $s_{1}-\mathrm{QS}$ we use 2.3. Thus assume that $a, b, x$ are distinct points in $R^{n}$ with $|b-x|=r,|a-x|=t r, t \leqq 2$. We must find an estimate

$$
|g(a)-g(x)| \leqq\left(t+s_{1}\right)|g(b)-g(x)|,
$$

where $s_{1}=s_{1}(q, n) \rightarrow 0$ as $q \rightarrow 0$.

Using the notation of $\left[\mathrm{TV}_{4}\right]$ we again obtain the estimate

$$
\left\|g-h_{Q}\right\|_{z_{Q}} \leqq M q \varrho_{Q}, \quad M=24 n^{2}
$$

$\left[\mathrm{TV}_{4},(5.9)\right]$. Here $Q$ is an arbitrary cube in $\mathscr{I}(p), h_{Q}: R^{p} \rightarrow Y$ is a similarity, and $\varrho_{Q}=L\left(h_{Q}\right) \lambda_{Q}$, where $\lambda_{Q}$ is the length of the side of $Q$. For $Q \in \mathscr{I}(p)$ set

$$
Y_{Q}^{\prime \prime}=\cup\left\{Y_{R}: Y_{R} \cap Y_{Q} \neq \emptyset\right\} .
$$

We may assume that $x \in R^{n} \backslash R^{p}$. Then there is $Q \in \mathscr{I}(p)$ such that $x \in Y_{Q}$. We divide the rest of the proof into two cases:

Case 1. $r \leqq \lambda_{Q} / 4$. Now

$$
|a-x| \leqq \lambda_{Q} / 2=d\left(Y_{Q}, R^{n} \backslash Y_{Q}^{\prime \prime}\right) .
$$

Hence $\{x, a, b\} \subset Y_{Q}^{\prime \prime}$. Let $W_{Q}^{\prime \prime}$ be the subcomplex of $W$ with $\left|W_{Q}^{\prime \prime}\right|=Y_{Q}^{\prime \prime}$. Let $\alpha_{0}=$ $\alpha_{0}\left(W_{Q}^{\prime \prime}\right)$ and $\Lambda=\Lambda\left(\alpha, W_{Q}^{\prime \prime}\right)$ be the numbers given by 2.14 . One can choose

$$
\alpha_{0}=\gamma_{0} \lambda_{Q}, \quad \Lambda\left(\alpha, W_{Q}^{\prime \prime}\right)=\Lambda_{0}\left(\alpha / \lambda_{Q}, n\right),
$$

for some $\gamma_{0}=\gamma_{0}(n)>0$ and for some function $\Lambda_{0}$ with $\lim _{\alpha \rightarrow 0} \Lambda_{0}(\alpha, n)=1$. Let $R$ be the unique cube in $\mathscr{I}(p)$ with $\lambda_{R}=2 \lambda_{Q}$ and $Q \subset R$. Then $Y_{Q}^{\prime \prime} \subset Z_{R}$. Hence (4.3) implies

$$
\left\|g-h_{R}\right\|_{Y_{Q}^{\prime \prime}} \leqq M q \varrho_{R}=\alpha L\left(h_{R}\right)
$$

with $\alpha=2 M q \lambda_{Q}$. We give the new restriction

$$
q \leqq \gamma_{0} / 2 M .
$$


Then $\alpha \leqq \alpha_{0}=\gamma_{0} \lambda_{Q}$, and Lemma 2.14 implies (4.2) with

$$
s_{1}=2\left(\Lambda_{0}(2 M q, n)^{2}-1\right) .
$$

Case 2. $r>\lambda_{Q} / 4$. Let $Q=R_{0} \subset R_{1} \subset \ldots$ be the unique sequence of cubes of $\mathscr{I}(p)$ such that $k\left(R_{j+1}\right)=k\left(R_{j}\right)+1$. Let $m$ be the smallest integer for which $Z_{R_{m}}$ contains $\{a, b\}$, and set $R=R_{m}$. Since $d\left(Z_{R_{j}}, R^{n} \backslash Z_{R_{j+1}}\right)=\lambda_{R_{j}}$, we have $r \geqq \lambda_{R} / 8$. From (4.3) we obtain

Hence

$$
\left\|g-h_{R}\right\|_{z_{R}} \leqq M q L\left(h_{R}\right) \lambda_{R} .
$$

$$
\begin{gathered}
|g(a)-g(x)| \leqq L\left(h_{R}\right)\left(t r+2 M q \lambda_{R}\right), \\
|g(b)-g(x)| \geqq L\left(h_{R}\right)\left(r-2 M q \lambda_{R}\right) .
\end{gathered}
$$

Assuming $q<1 / 16 M$ we obtain

$$
\frac{|g(a)-g(x)|}{|g(b)-g(x)|} \leqq \frac{t+16 M q}{1-16 M q}
$$

which implies (4.2).

4.4. Corollary. Let $Y$ be a linear subspace of $l_{2}$, and let $p \leqq n \leqq \operatorname{dim} Y$. Then a set $A \subset R^{p}$ has the BLEP or the QSEP in $\left(R^{p}, Y\right)$ if and only if it has the same property in $\left(R^{n}, Y\right)$. In particular, the extension properties in $R^{n}$ and in $\left(R^{p}, R^{n}\right)$ are equivalent for $A \subset R^{p}$.

It is natural to ask whether $R^{n}$ has the extension properties in $l_{2}$. I do not know the answer. However, the following result in this direction can be established:

4.5. Theorem. Every L-bilipschitz $f: R^{n} \rightarrow R^{n}$ can be extended to an L-bilipschitz homeomorphism $g: l_{2} \rightarrow l_{2}$, and every $s-\mathrm{QS} f: R^{n} \rightarrow R^{n}$ can be extended to an $s_{1}-\mathrm{QS}$ homeomorphism $g: l_{2} \rightarrow l_{2}$ such that $s_{1}=s_{1}(s, n) \rightarrow 0$ as $s \rightarrow 0$. Moreorer, $g Y=Y$ for every linear subspace $Y$ of $l_{2}$ containing $R^{n}$.

Proof. Let $E$ be the orthogonal complement of $R^{n}$ in $l_{2}$. The bilipschitz case is easy; we define $g(x+y)=f(x)+y$ for $x \in R^{n}, y \in E$.

Suppose that $f: R^{n} \rightarrow R^{n}$ is $s-\mathrm{QS}$. Then $f$ is $K-\mathrm{QC}$ with $K=K(s, n)$. By $\left[\mathrm{TV}_{3}\right], f$ can be extended to a homeomorphism $F: R_{+}^{n+1} \rightarrow R_{+}^{n+1}$ such that $F \mid$ int $R^{n+1}$ is $H$-bilipschitz in the hyperbolic metric with $H=H(s, n)$. The required homeomorphism $g$ is then the rotation of $F$ around $K^{n}$. More precisely, let $e \in E$ be a unit vector. If $x \in R^{n}$ and $t>0$, we define $g(x+t e)=x^{\prime}+t^{\prime} e$, where $\left(x^{\prime}, t^{\prime}\right)$ is determined by $x^{\prime}+t^{\prime} e_{n+1}=F\left(x+t e_{n+1}\right)$. If $a, b, x$ are points in $l_{2}$, there is a linear subspace $Y$ of $l_{2}$ with $\operatorname{dim} Y=n+3$ containing these points and $R^{n}$. Arguing as in $\left[\mathrm{TV}_{3}\right.$, 3.13], we see that $g$ defines a $K_{1}-\mathrm{QC}$ map $g_{1}: Y \rightarrow Y$ with $K_{1}=K_{1}(s, n)$. Hence $g_{1}$ is $s_{1}-\mathrm{QS}$ with $s_{1}=s_{1}(s, n)$. Herc $: g$ is $s_{1}-\mathrm{QS}$.

If $s$ is small, the extension $F$ of $f$ can also be obtained from the fact that $R^{n}$ has the QSEP in $R^{n+1}$. Then $F$ is $s_{2}-\mathrm{QS}$ with small $s_{2}$. However, we need the fact that the 
hyperbolic bilipschitz constant $H$ of $F$ int $R^{n+1}$ is close to 1 . This follows rather easily from the proof of $\left[\mathrm{TV}_{4}, 5.4\right]$. This implies that $s_{1}(s, n) \rightarrow 0$ as $s \rightarrow 0$.

4.6. Theorem. If $Y$ is a linear supspace of $l_{2}$ and if $p<n \leqq \operatorname{dim} Y$, then $S^{p}, R_{+}^{p+1}$ and $\bar{B}^{p+1}$ have the extension properties in $\left(R^{n}, Y\right)$.

Proof. The case $p=0$ needs a separate argument, which is omitted. Assume $p \geqq 1$. The case $A=S^{p}$ follows from 4.1 by means of auxiliary inversions as in $\left[\mathrm{TV}_{4}, 5.23\right]$. The awkward proof of $\left[\mathrm{IV}_{4}, 5.22\right]$ can be essentially simplified by means of quasimöbius maps, see [ $\left.\mathrm{Vä}_{2}, 3.11\right]$.

The case $A=R_{+}^{p+1}$ can be proved by modifying the proof of 4.1. By 4.4, we may assume $n=p+1$. If $f: R_{+}^{n} \rightarrow Y$ is $L$-bilipschitz or $s-\mathrm{QS}$ with small $L$ or $s$, we define an extension $g_{0}: R_{-}^{n} \rightarrow Y$ of $f \mid R^{p}$ as in the proof of 4.1. However, when defining the orthogonal frames $u_{Q} \in V_{n}^{0}(Y)$, we do not make use of the results of [ $\left[\mathrm{V}_{4}\right.$, Section 4]. Instead, we can now define $w_{Q}^{j}=f\left(a_{Q}+\lambda_{Q} e_{j}\right)-f\left(a_{Q}\right)$ also for $j=n$, and we let $u_{Q}$ be the Gram-Schmidt orthogonalization of $w_{Q}=\left(w_{Q}^{1}, \ldots, w_{Q}^{n}\right)$. We obtain an extension $g: R^{n} \rightarrow Y$ of $f$. We still have to show that $g$ is $L_{1}$-bilipschitz or $s_{1}-$ QS. It follows from the proof of 4.1 that it suffices to show that

$$
\left\|f-h_{Q}\right\|_{Z_{Q}^{+}} \leqq 24 n^{2} q \varrho_{Q}
$$

for sufficiently small $L$ or $s$, where $Z_{Q}^{+}=Z_{Q} \cap R_{+}^{n}$. This follows rather easily from a slightly modified version of $\left[\mathrm{TV}_{4}, 3.10\right]$. We omit the details.

Finally, the case $A=\bar{B}_{+}^{p+1}$ follows from the preceding case by auxiliary inversions. Alternatively, it is a special case of 6.13.1.

\section{The first condition}

In Theorem 5.5 we shall give a sufficient condition for a set $A \subset R^{n}$ to have the extension properties in $R^{n}$. We then show that this condition holds for all compact $(n-1)$-dimensional DIFF and PL manifolds and for certain other sets in $R^{n}$.

5.1. The Whitney triangulation. Let $G \subset R^{n}$ be an open set, $\emptyset \neq G \neq R^{n}$. The relative size of a compact set $A \subset G$ is defined as

$$
r_{G}(A)=\frac{d(A)}{d(A, \partial G)} .
$$

Let $K$ be the Whitney decomposition of $G$ into closed $n$-cubes such that

$$
\lambda_{1} \leqq r_{G}(Q) \leqq \lambda_{2}
$$

for all $Q \in K$, where $\lambda_{1}$ and $\lambda_{2}$ are positive constants. See e.g. [St, p. 167] or [TV 7.2]. One can choose $\lambda_{1}=1 / 7$ and $\lambda_{2}=\sqrt{n} / 2$, but these constants can obviously be chosen to be arbitrarily small. 
We define a subdivision of $K$ to a simplicial complex $W$ as follows: Suppose that we have defined a simplicial subdivision $W^{p}$ of the $p$-skeleton $K^{p}$ of $K$. Let $Q$ be a $(p+1)$-cube of $K$, and let $v_{Q}$ be the center of $Q$. Since $\partial Q$ is the underlying space of a subcomplex $L_{Q}$ of $W^{p}$, the cone construction $v_{Q} L_{Q}$ gives a simplicial subdivision of $Q$, and we obtain $W^{p+1}$. The complex $W$ is called a Whitney triangulation of $G$.

If $\sigma$ is an $n$-simplex of $W$, we can write

$$
\varrho(\sigma) \leqq \varrho_{n}, \quad a_{1} \leqq r_{G}(\sigma) \leqq a_{2},
$$

where the numbers $\varrho_{n}, a_{1}, a_{2}$ depend only on $n$. Indeed, since the simplexes of $W$ belong to a finite number of similarity classes, the first inequality of (5.2) is true. In the second one, we can choose $a_{1}=\lambda_{1} / 3 \sqrt{n}$ and $a_{2}=\lambda_{2} / 2$.

5.3. Terminology. Let $A \subset R^{n}$. We say that a simplex $\Delta$ is a simplex of $A$ if $\Delta^{0} \subset A$. If $\Delta$ is a an $n$-simplex of $A$ and if $f: A \rightarrow R^{n}$ is a map, we say that $f \mid \Delta^{0}$ is sense-preserving if the unique affine extension $g: R^{n} \rightarrow R^{n}$ of $f \mid \Delta^{0}$ is sense-preserving. Two $p$-simplexes $\Delta, \Delta^{\prime}$ of $A$ are said to be $M$-related in $A, M \geqq 1$, if there is a finite sequence $\Delta=\Delta_{0}, \ldots, \Delta_{k}=\Delta^{\prime}$ of $p$-simplexes of $A$ such that

(1) $\varrho\left(\Delta_{\jmath}\right) \leqq M$ for $0 \leqq j \leqq k$,

(2) $1 / M \leqq d\left(\Delta_{j}\right) / d\left(\Delta_{j-1}\right) \leqq M$ for $1 \leqq j \leqq k$,

(3) $d\left(\Delta_{j-1}, \Delta_{j}\right) \leqq M \min \left(d\left(\Delta_{j-1}\right), d\left(\Delta_{j}\right)\right)$ for $1 \leqq j \leqq k$.

5.4. Lemma. Let $n$ be a positive integer, let $M \geqq 1$, and let $s=s(M, n)$ be such that $\varkappa(s, n) \leqq 1 / 10 M^{3}(n+1)$, where $\varkappa$ is the function of 3.1. Suppose that $A \subset R^{n}$, that $f: A \rightarrow R^{n}$ is $s-\mathrm{QS}$ and that the $n$-simplexes $\Delta_{1}, \Delta_{2}$ of $A$ are $M$-related in $A$. Then $f \mid \Delta_{1}^{0}$ and $f \mid \Delta_{2}^{0}$ are either both sense-preserving or both sense-reversing.

Proof. We may assume that the sequence $\Delta_{0}, \ldots, \Delta_{k}$ of 5.3 is the pair $\left(\Delta_{1}, \Delta_{2}\right)$. Suppose that $f \mid \Delta_{1}^{0}$ is sense-preserving. Set

$$
F=\left\{x \in A: d\left(x, \Delta_{1}\right) \leqq 2 M d\left(\Delta_{1}\right)\right\} .
$$

Then $d(F) \leqq 5 M d\left(\Delta_{1}\right)$. For every $z \in \Delta_{2}$ we have

$$
d\left(z, \Delta_{1}\right) \leqq d\left(\Delta_{2}\right)+d\left(\Delta_{2}, \Delta_{1}\right) \leqq 2 M d\left(\Delta_{1}\right) .
$$

Hence $\Delta_{2}^{0} \subset F$. Applying 3.1 we choose a similarity $h: R^{n} \rightarrow R^{n}$ such that

$$
\|h-f\|_{F} \leqq \chi(s, n) L_{h} d(F) \leqq \frac{L_{h} d\left(\Delta_{1}\right)}{2(n+1) M^{2}} .
$$

Since $\varrho\left(\Delta_{1}\right) \leqq M \leqq M^{2}$, we have

$$
|f(z)-h(z)| \leqq \frac{L_{h} d\left(\Delta_{1}\right)}{2(n+1) \varrho\left(\Delta_{1}\right)}
$$


for every $z \in \Delta_{1}^{0}$. By $2.7, h$ is sense-preserving. Furthermore, since $d\left(\Delta_{1}\right) \leqq M d\left(\Delta_{2}\right)$, we have for every $y \in \Delta_{2}^{0}$,

$$
|f(y)-h(y)| \leqq \frac{L_{h} d\left(\Delta_{2}\right)}{2(n+1) \varrho\left(\Delta_{2}\right)} .
$$

Again by 2.7, $f \mid \Delta_{2}^{0}$ is sense-preserving.

5.5. Theorem. Suppose that $n \geqq 2$, that $A$ is closed in $R^{n}$, that $\partial A$ is bounded and that int $A$ has a finite number of components. For $x \in R^{n} \backslash A$ and $b>1$ we set

$$
E(x, b)=A \cap \bar{B}(x, b d(x, A)) .
$$

Suppose that there exist numbers $b_{2} \geqq b_{1}>1, M \geqq 1$, and that for every $\lambda>0$ there is $r_{0}>0$ such that if $x \in R^{n} \backslash A$ and $d(x, A)=r \leqq r_{0}$, then one of the following two conditions is satisfied:

(a) There is an (n-1)-simplex $\Delta$ of $E\left(x, b_{1}\right)$ and an (n-1)-plane $T \subset R^{n}$ such that

$\left(\mathrm{a}_{1}\right) \quad \varrho(\Delta) \leqq M$,

( $\left.\mathrm{a}_{2}\right) \quad d(\Delta) \geqq r / M$,

(a) $E\left(x, b_{1}\right) \subset T+\lambda r \bar{B}^{n}$.

(b) There is an n-simplex $\Delta$ of $E\left(x, b_{2}\right)$ such that

$\left(b_{1}\right) \quad d(\Delta) \geqq r / M$,

$\left(\mathrm{b}_{2}\right) \Delta$ is $M$-related to an $n$-simplex $\Delta^{\prime}$ in $A$ with $d\left(\Delta^{\prime}\right) \geqq 1 / M$.

Then $A$ has the extension properties in $R^{n}$.

Proof. Choose an auxiliary parameter $q>0$. To prove the QSEP, it suffices to show that there are $q_{0}>0$ and for every $q \in\left(0, q_{0}\right]$ a number $s=s(q, A, n)>0$ such that every $s-$ QS embedding $f: A \rightarrow R^{n}$ has an extension to a $K-$ QC map $g: R^{n} \rightarrow$ $R^{n}$, where $K=K(q, A, n) \rightarrow 1$ as $q \rightarrow 0$. In the bilipschitz case, we find $L=L(q, A, n)$ such that every $L$-bilipschitz map $f: A \rightarrow R^{n}$ has an extension to an $L_{1}$-bilipschitz $g: R^{n} \rightarrow R^{n}$ with $L_{1}=L_{1}(q, A, n) \rightarrow 1$ as $q \rightarrow 0$.

To begin with, we only assume $0<q \leqq 1$. In the course of the proof, we shall give more restrictions on $q$ of the form $q \leqq q_{0}(A, n)$.

Choose $R \geqq 4$ such that $\partial A \subset B^{n}\left(R / 2 b_{2}\right)$, and set $B=\bar{B}^{n}(R)$. Next choose $r_{1}>0$ such that every component of int $A$ contains a ball $B\left(x, r_{1}\right) \subset B$. Set $\lambda=q / 4$ and choose the corresponding $r_{0}$. We may assume $r_{0} \leqq 1$. Let $x$ be the function given by 3.1. Choose $s=s(q, A, n) \in(0, q]$ such that $x(s, n)$ is smaller than the numbers

$$
\frac{1}{10 R M^{3} b_{2}(n+1)}, \frac{q r_{0}}{2 R}, \frac{q}{4 b_{2}}, \frac{r_{1}}{5 R} .
$$

We show that $s$ is the required number provided that $q$ is sufficiently small. In the 
bilipschitz case, we set $L=L(q, A, n)=\left(s^{2}+1\right)^{1 / 2}$. Then every $L$-bilipschitz map is $s-\mathrm{QS}$.

Suppose that $f: A \rightarrow R^{n}$ is $s-\mathrm{QS}$. By 3.1, there is a similarity $h$ of $R^{n}$ such that

$$
\|h-f\|_{A \cap B} \leqq \varkappa(s, n) L_{h} d(A \cap B) \leqq 2 R L_{h} \varkappa(s, n) .
$$

Replacing $f$ by $h^{-1} f$ we may assume that

$$
\|f-\mathrm{id}\|_{A \cap B} \leqq 2 R \varkappa(s, n) .
$$

Set

$$
G=R^{\eta} \backslash A, \quad G\left(r_{0}\right)=\left\{x \in G: d(x, A)<r_{0}\right\} .
$$

Let $G_{1}$ be the set of all points $x \in G\left(r_{0}\right)$ which satisfy the condition (a), and set $G_{2}=$ $G\left(r_{0}\right) \backslash G_{1}$. For $x \in G$ we define

$$
\begin{array}{lll}
E_{x}=E\left(x, b_{1}\right) & \text { if } & x \in G_{1}, \\
E_{x}=E\left(x, b_{2}\right) & \text { if } & x \in G \backslash G_{1} .
\end{array}
$$

We associate to every $x \in G$ a similarity $h_{x}$ of $R^{n}$ as follows: If $x \in G \backslash G\left(r_{0}\right)$, we choose $h_{x}=$ id. Assume $x \in G\left(r_{0}\right)$. Then $d(x, A)=r<r_{0}$. If $x \in G_{1}$, we apply 3.1 to find a similarity $k_{x}$ such that

$$
\left\|k_{x}-f\right\|_{E_{x}} \leqq \chi(s, n) L\left(k_{x}\right) d\left(E_{x}\right) \leqq 2 b_{1} r L\left(k_{x}\right) \chi(s, n) .
$$

If $k_{x}$ is sense-preserving, we choose $h_{x}=k_{x}$. Otherwise, we set $h_{x}=k_{x} \psi$, where $\psi$ is the reflection in the $(n-1)$-plane $T$ given by (a). Finally, if $x \in G_{2}$, we again apply 3.1 and choose $h_{x}$ so that

$$
\left\|h_{x}-f\right\|_{E_{x}} \leqq \varkappa(s, n) L\left(h_{x}\right) d\left(E_{x}\right) \leqq 2 b_{2} r L\left(h_{x}\right) \chi(s, n) .
$$

Now $h_{x}$ is defined for all $x \in G$. In the bilipschitz case, $h_{x}$ is chosen to be an isometry.

We next show that $h_{x}$ is sense-preserving for every $x \in G$. For $x \in G \backslash G_{2}$, this follows directly from the construction. Suppose $x \in G_{2}$. Let $\Delta$ and $\Delta^{\prime}$ be the $n$-simplexes of $A$ given by (b), and let $\Delta=\Delta_{0}, \ldots, \Delta_{k}=\Delta^{\prime}$ be the sequence given by the definition 5.3 of $M$-relatedness, We first show that one can choose $\Delta^{\prime}$ to be a simplex of $A \cap B$. If $A$ is bounded, $A \subset B$, and this is trivial. Assume that $A$ is unbounded. Then $A$ contains $R^{n} \backslash B^{n}(R / 2)$. If all vertices of $\Delta^{\prime}$ are in $R^{n} \backslash B^{n}(R / 2)$, we can continuously deform $\Delta^{\prime}$ in $R^{n} \backslash B^{n}(R / 2)$ to a simplex $\Delta^{\prime \prime}$ of $B \backslash B^{n}(R / 2)$ with $d\left(\Delta^{\prime \prime}\right)=$ $R / 4 \geqq 1 \geqq 1 / M$ without changing its similarity class. Thus $\Delta$ is $M$-related to $\Delta^{\prime \prime}$ in $A$. If $\Delta^{\prime}$ has vertices both in $B^{n}(R / 2)$ and $R^{n} \backslash B$, we choose a translation $\varphi$ of $R^{n}$ such that $\varphi \Delta^{\prime} \cap B^{n}(R / 2)=\emptyset \neq \varphi \Delta^{\prime} \cap \Delta^{\prime}$. Then the sequence $\Delta_{0}, \ldots, \Delta_{k}, \varphi \Delta^{\prime}$ still satisfies the conditions of 5.3, and the situation reduces to the preceding case.

Since $x(s, n) \leqq 1 / 4(n+1) R M^{2}$ and since $b\left(\Delta^{\prime}\right)=d\left(\Delta^{\prime}\right) / \varrho\left(\Delta^{\prime}\right) \geqq 1 / M^{2}$, implies

$$
|f(x)-x| \leqq \frac{b\left(\Delta^{\prime}\right)}{2(n+1)}
$$


for every vertex $x$ of $\Delta^{\prime}$. By $2.7, f^{\prime}\left(\Delta^{\prime}\right)^{0}$ is sense-preserving. Hence, by Lemma 5.4 and by (5.6), $f \mid \Delta^{0}$ is sense-preserving. Since $b(\Delta) \geqq r / M^{2}, 2.7,(5.6)$ and (5.9) imply that $h_{x}$ is sense-preserving.

We next prove the inequality

$$
\left\|h_{x}-f\right\|_{E_{x}} \leqq q d(x, A) L\left(h_{x}\right)
$$

for every $x \in G$. Set $r=d(x, A)$. We divide the proof into four cases.

Case 1. $r \geqq r_{0}$. If $A$ is not bounded, $G \subset B^{n}\left(R / 2 b_{2}\right)$. Hence $r \leqq R / 2 b_{2}$, which implies $E_{x} \subset A \cap B$. This is clearly also true if $A$ is bounded. Since $h_{x}=\mathrm{id}$ and since $\chi(s, n) \leqq q r_{0} / 2 R$, we obtain

$$
\left\|h_{x}-f\right\|_{E_{x}} \leqq\|\mathrm{id}-f\|_{A \cap B} \leqq 2 R \varkappa(s, n) \leqq q r L\left(h_{x}\right) .
$$

Case 2. $r<r_{0}, x \in G_{1}, h_{x}=k_{x}$. Now (5.10) follows from (5.8) and from the inequality $\chi(s, n) \leqq q / 4 b_{2}<q / 2 b_{1}$.

Case 3. $r<r_{0}, x \in G_{1}, h_{x}=\psi k_{x}$. Now $L\left(h_{x}\right)=L\left(k_{x}\right)$. For every $y \in E_{x},(5.8)$ yields

$$
\begin{aligned}
\left|h_{x}(y)-f(y)\right| & \leqq\left|k_{x}(\psi(y))-k_{x}(y)\right|+\left|k_{x}(y)-f(y)\right| \\
& \leqq 2 L\left(h_{x}\right) \lambda r+2 b_{1} r L\left(h_{x}\right) \varkappa(s, n) .
\end{aligned}
$$

Since $\lambda=q / 4$ and since $\chi(s, n) \leqq q / 4 b_{2} \leqq q / 4 b_{1}$, we obtain (5.10).

Case 4. $r<r_{0}, x \in G_{2}$. Since $\varkappa(s, n) \leqq q / 4 b_{2}<q / 2 b_{2}$, this case follows from (5.9). Thus (5.10) is proved.

Choose a Whitney triangulation $W$ of $G$ satisfying (5.2). Here we choose

$$
a_{2} \leqq \min \left(1,\left(b_{1}-1\right) / 2\right) \text {. }
$$

The constants $a_{1}$ and $a_{2}$ depend only on $A$ and $n$.

For every vertex $v$ of $W$ we set

$$
g(v)=h_{v}(v),
$$

and extend $g$ affinely to every simplex of $W$. Setting $g \mid A=f$ we obtain a map $g: R^{n} \rightarrow$ $R^{n}$. We claim that $g$ is the desired extension of $f$.

We first show that $g$ is continuous. This is clearly true in $G$ and in int $A$. Suppose that $x_{0} \in \partial A=\partial G$, and let $\varepsilon>0$. Since $f$ is continuous, there is $\delta>0$ such that $\left|f(x)-f\left(x_{0}\right)\right| \leqq \varepsilon$ whenever $x \in A$ and $\left|x-x_{0}\right| \leqq \delta$. Choose $\delta_{1}<\delta$ such that $E_{v} \subset$ $B\left(x_{0}, \delta\right)$ and $d(v, A) \leqq r_{0}$ whenever $v$ is a vertex of any $n$-simplex $\sigma \in W$ such that $d\left(x_{0}, \sigma\right) \leqq \delta_{1}$. Suppose that $x \in G$ with $\left|x-x_{0}\right| \leqq \delta_{1}$. Choose an $n$-simplex $\sigma \in W$ containing $x$. It suffices to find an estimate

$$
\left|g(v)-f\left(x_{0}\right)\right| \leqq M_{1} \varepsilon
$$

for the vertices $v$ of $\sigma$ with some constant $M_{1}$. In what follows, we let $M_{2}, M_{3}, \ldots$ denote constants $M_{j} \geqq 1$ depending only on $A$ and $n$. Set $r=d(v, A)$, and choose 
$y \in A$ with $|y-v|=r$. Since $q \leqq 1,(5.10)$ implies

$$
\begin{aligned}
\left|g(v)-f\left(x_{0}\right)\right| & \leqq\left|h_{v}(v)-h_{v}(y)\right|+\left|h_{v}(y)-f(y)\right|+\left|f(y)-f\left(x_{0}\right)\right| \\
& \leqq L\left(h_{v}\right) r+q r L\left(h_{v}\right)+\varepsilon \\
& \leqq 2 r L\left(h_{v}\right)+\varepsilon .
\end{aligned}
$$

Since $r \leqq r_{0}, E_{v}$ contains points $x_{1}, x_{2}$ with $\left|x_{1}-x_{2}\right| \geqq r / M$. We give the restriction $q \leqq 1 / 4 M$. Then (5.10) implies

$$
\begin{aligned}
r L\left(h_{v}\right) / M & \leqq L\left(h_{v}\right)\left|x_{1}-x_{2}\right|=\left|h_{v}\left(x_{1}\right)-h_{v}\left(x_{2}\right)\right| \\
& \leqq\left|f\left(x_{1}\right)-f\left(x_{2}\right)\right|+2 q r L\left(h_{v}\right) \\
& \leqq 2 \varepsilon+r L\left(h_{v}\right) / 2 M,
\end{aligned}
$$

and hence $r L\left(h_{v}\right) \leqq 4 M \varepsilon$. This implies (5.11) with $M_{1}=8 M+1$ and proves the continuity of $g$.

Let $\sigma$ be an $n$-simplex of $W$, and let $v$ be the vertex of $\sigma$ which is closest to $A$. We want to estimate $\left|h_{v}-g\right|$ in $\sigma^{0}$. Set $r=d(v, A)$. If $r \geqq r_{0}, h_{v}=\mathrm{id}=g$ in $\sigma^{0}$. Assume that $r<r_{0}$. Set

$$
c_{1}=\frac{b_{1}-1}{2\left(b_{2}-1\right)}, \quad r^{\prime}=c_{1} r .
$$

Then $c_{1}$ depends only on $A$, and

$$
\frac{a_{2}}{b_{2}-1} \leqq c_{1} \leqq \frac{1}{2}
$$

Choose $y \in A$ with $|y-v|=r$. Let $z$ be the unique point on the segment $v y$ such that $|z-y|=r^{\prime}$. A direct computation shows

$$
|v-z|+b_{2} r^{\prime}=r\left(1+b_{1}\right) / 2 .
$$

Moreover, $r^{\prime}=d(z, A)$. Let $x \in E\left(z, b_{2}\right)$. If $u \in \sigma^{0}$, then

Hence (5.12) gives

$$
|u-v| \leqq d(\sigma) \leqq a_{2} d(\sigma, A) \leqq\left(b_{1}-1\right) r / 2 .
$$

$$
|x-u| \leqq|x-z|+|z-v|+|v-u| \leqq b_{1} r \leqq b_{1} d(u, A) .
$$

Thus

$$
E\left(z, b_{2}\right) \subset A \cap \bar{B}\left(u, b_{1} r\right) \subset E\left(u, b_{1}\right) \subset E_{u} .
$$

Since $r^{\prime}<r_{0}$, there is an $(n-1)$-simplex $\Delta$ of $E\left(z, b_{2}\right)$ such that $\varrho(\Delta) \leqq M$ and $d(\Delta) \geqq r^{\prime} / M$. If $z \in G_{2}, \Delta$ is a suitable face of the $n$-simplex given by (b). Since $d(u, A) \leqq\left(1+a_{2}\right) r \leqq 2 r,(5.10)$ and (5.13) yield for every $x \in \Delta^{0}$ :

$$
\begin{aligned}
\left|h_{v}(x)-h_{u}(x)\right| & \leqq\left|h_{v}(x)-f(x)\right|+\left|f(x)-h_{u}(x)\right| \\
& \leqq q r L\left(h_{v}\right)+2 q r L\left(h_{u}\right) .
\end{aligned}
$$


We give the new restriction $q \leqq c_{1} / 8 M$. Since $r \leqq M d(\Delta) / c_{1}$, (5.10) and (5.13) imply

$$
\begin{aligned}
L\left(h_{u}\right) d(\Delta) & =d\left(h_{u} \Delta\right) \leqq d\left(f \Delta^{0}\right)+2\left\|h_{u}-f\right\|_{E_{u}} \\
& \leqq d\left(f \Delta^{0}\right)+4 q r L\left(h_{u}\right) \\
& \leqq d\left(f \Delta^{0}\right)+L\left(h_{u}\right) d(\Delta) / 2 .
\end{aligned}
$$

Since $\Delta^{0} \subset E_{v}$, this yields

Hence (5.14) gives

$$
\begin{aligned}
L\left(h_{u}\right) d(\Delta) & \leqq 2 d\left(f \Delta^{0}\right) \leqq 2 d\left(h_{v} \Delta\right)+4\left\|h_{v}-f\right\|_{E_{v}} \\
& \leqq 2 L\left(h_{v}\right) d(\Delta)+4 q r L\left(h_{v}\right)<3 L\left(h_{v}\right) d(\Delta) .
\end{aligned}
$$

$$
\left\|h_{v}-h_{u}\right\|_{\Delta^{0}} \leqq 7 q r L\left(h_{v}\right) \text {. }
$$

By (5.13), $|x-u| \leqq b_{1} r$ for every $x \in \Delta^{0}$. Since $\varrho(\Delta) \leqq M$ and $d(\Delta) \geqq c_{1} r / M, 2.12$ yields

Furthermore,

$$
\left|h_{v}(u)-g(u)\right| \leqq M_{2} q r L\left(h_{v}\right) .
$$

$$
r \leqq d(\sigma)+d(\sigma, A) \leqq\left(1+a_{1}^{-1}\right) \varrho_{n} b(\sigma) .
$$

We set $M_{3}=2\left(1+a_{1}^{-1}\right) \varrho_{n}(n+1) M_{2}$ and give the new restriction $q \leqq 1 / M_{3}$. Then 2.7 implies that $g \mid \sigma$ is sense-preserving and that

$$
\begin{gathered}
L(g \mid \sigma) \leqq L\left(h_{v}\right)\left(1+M_{3} q\right), \quad l(g \mid \sigma) \geqq L\left(h_{v}\right) /\left(1+M_{3} q\right), \\
H(g \mid \sigma) \leqq\left(1+M_{3} q\right)^{2} .
\end{gathered}
$$

In the bilipschitz case $L\left(h_{v}\right)=1$, and hence $g \mid \sigma$ is $\left(1+M_{3} q\right)$-bilipschitz.

We use degree theory to show that $g$ is a homeomorphism onto $R^{n}$. The topological degree $\mu(y, f, D)$ is an integer defined whenever $D$ is a bounded domain in $R^{n}$, $f: \bar{D} \rightarrow R^{n}$ is continuous, and $y \in R^{n} \backslash f \partial D$; see e.g. [Do, IV. 5] or [RR, II. 2]. If $G \subset R^{n}$ is open and if $f: G \rightarrow R^{n}$ is continuous, $f$ is said to be sense-preserving if $\mu(y, f, D)>0$ whenever $\bar{D}$ is compact in $G$ and $y \in f D \backslash f \partial D$.

We first show that $g \mid$ int $A=f \mid$ int $A$ is sense-preserving. Let $V$ be a component of int $A$. Then there is a ball $B_{V}=B\left(x_{V}, r_{1}\right) \subset V \cap B$. By (5.6), $\varkappa(s, n) \leqq r_{1} / 5 R$, and therefore

$$
\|f-\mathrm{id}\|_{B_{V}} \leqq 2 R \varkappa(s, n)<r_{1} / 2 .
$$

Consequently, the segmental homotopy $h_{t}: f \simeq$ id satisfies $h_{t}\left(x_{V}\right) \notin h_{t} \partial B_{V}$, and thus

$$
\mu\left(f\left(x_{V}\right), f, B_{V}\right)=\mu\left(x_{V}, \text { id, } B_{V}\right)=1 .
$$

Since $f \mid V$ is an embedding, $f$ int $A$ is sense-preserving.

We next show that $g$ is sense-preserving. Let $D \subset R^{n}$ be a bounded domain, and let $y \in g D \backslash g \partial D$. Set $Y=\partial A \cup\left|W^{n-1}\right|$, where $W^{n-1}$ is the $(n-1)$-skeleton of $W$. Then int $Y=\emptyset$. Since $g \mid \partial A$ is an embedding and since $g \mid G$ is PL, we have int $g Y=$ $\emptyset$. Let $U$ be the $y$-component of $R^{n} \backslash g \partial D$. Then $D_{0}=D \cap g^{-1} U$ is open and nonempty, and so is $D_{0} \backslash Y$. Since $g \mid R^{n} \backslash Y$ is an immersion, $g\left[D_{0} \backslash Y\right]$ is open. Hence we can choose a point $z \in g D_{0} \backslash g Y$. Since $z \in U, \mu(y, g, D)=\mu(z, g, D)$. On the other 
hand, $D \cap g^{-1}(z)$ is a finite nonempty subset of $D \backslash Y$, and $g \mid D \backslash Y$ is a sense-preserving immersion. Hence

$$
\mu(z, g, D)=\operatorname{card}\left(D \cap g^{-1}(z)\right)>0,
$$

which implies that $g$ is sense-preserving.

Clearly each fiber $g^{-1}(y)$ is countable. Consequently, $g$ is light and sense-preserving, hence discrete and open [TY, Corollary, p. 333]. Furthermore, $g \mid R^{n} \backslash B$ is a homeomorphism onto a neighborhood of $\infty$. Indeed, if $A$ is bounded, $g \mid R^{n} \backslash B=$ id. If $A$ is unbounded, $g\left|R^{n} \backslash B=f\right| R^{n} \backslash B$ is a QS embedding, and $g(x) \rightarrow \infty$ as $x \rightarrow \infty$. Hence there is a ball $B_{1}=B^{n}\left(R_{1}\right)$ containing $B$ such that $g B \cap g\left[R^{n} \backslash B_{1}\right]=\emptyset$. Let $V$ be the bounded component of $R^{n} \backslash g \partial B_{1}$. Then $\mu\left(y, g, B_{1}\right)=k$ is independent of $y \in V$. Choosing $y \in V \backslash g B$ we see $k=1$. Hence we obtain for every $y \in V$

$$
1=\mu\left(y, g, B_{1}\right)=\Sigma\left\{i(x, g): x \in B_{1} \cap g^{-1}(y)\right\} \geqq \operatorname{card}^{-1}(y),
$$

where $i(x, g)$ is the local degree of $g$ at $x$. Thus $g$ is a homeomorphism onto $R^{n}$.

In the bilipschitz case, it follows from (5.15) that $g$ is $L_{1}$-bilipschitz with $L_{1}=$ $\max \left(L, 1+M_{3} q\right)$. In the QS case, it follows from (5.15) and from a standard removability theorem [ $\left.V \ddot{a}_{1}, 35.1\right]$ that $g \mid G$ is $\left(1+M_{3} q\right)^{2 n-2}-\mathrm{QC}$. If $\partial A$ is of $\sigma$-finite $(n-1)$ measure, $\left[V \ddot{a}_{1}, 35.1\right]$ implies that $g$ is $K-\mathrm{QC}$ with

$$
K=\max \left((1+s)^{n-1},\left(1+M_{3} q\right)^{2 n-2}\right),
$$

and thus $A$ has the QSEP. Since this case is sufficient in the applications 5.17 and 5.19 , and since a detailed proof of the general case would take several pages, we only give a sketch of it.

To show that $g$ is QC, it suffices to find a uniform upper bound for the metric dilatation $H(x, g)$, see $\left[V \ddot{a}_{1}, 34.1\right]$. Once this has been done, the desired estimate for the dilatation of $g$ is easily obtained by considering the derivative of $g$ at points of density of $\partial A$.

Let $z \in \partial A$ and $x \in G$ with $|x-z|=r$, where $r$ is small. Choose a suitable $c_{2}>1$ and apply 3.1 to find a similarity $h$ such that $|h(y)-f(y)| \leqq M_{4} q L_{h} r$ for $y \in A \cap$ $B\left(z, c_{2} r\right)$. It suffices to find $M_{5}$ such that

$$
L_{h} r / M_{5} \leqq|g(x)-f(z)| \leqq M_{5} L_{h} r .
$$

The second inequality is fairly easy. With a small loss of generality, assume $x \in W^{0}$. Let $r_{1}=d(x, A)$ and choose $y \in A$ with $|y-x|=r_{1}$. We may assume that $E_{x} \subset A \cap B\left(z, c_{2} r\right)$. Then

$$
\begin{aligned}
|g(x)-f(z)| & \leqq\left|h_{x}(x)-h_{x}(y)\right|+\left|h_{x}(y)-f(y)\right|+|f(y)-h(y)| \\
& +|h(y)-h(z)|+|h(z)-f(z)| \\
& \leqq L\left(h_{x}\right) r_{1}+L\left(h_{x}\right) q r_{1}+2 M_{4} q L_{h} r+c_{2} L_{h} r .
\end{aligned}
$$

Thus it suffices to show that $L\left(h_{x}\right) r_{1} \leqq M_{6} L_{h} r$. For this, observe that $E_{x}$ contains 
a point $a$ with $r_{1} \leqq 2 M|a-y|$. Then

$$
L\left(h_{x}\right)|a-y|=\left|h_{x}(a)-h_{x}(y)\right| \leqq|f(a)-f(y)|+4 L\left(h_{x}\right) q M|a-y| .
$$

Since we may assume that $q \leqq 1 / 8 M$, this gives

and hence

$$
L\left(h_{x}\right)|a-y| \leqq 2|f(a)-f(y)| \leqq 2 L_{h}|a-y|+4 M_{4} q L_{h} r,
$$

$$
L\left(h_{x}\right) r_{1} \leqq\left(2+M_{4}\right) L_{h} r .
$$

The first inequality of (5.16) is harder. We first replace $b_{1}$ by $b_{1}^{\prime}=$ $\max \left(b_{1}, 2\left(2+a_{1}\right)\left(1+a_{1}\right)\right)$ and show that this is no loss of generality. Choose $\sigma$ with $x \in \sigma \in W$. Consider a vertex $v$ of $\sigma$, set $\alpha r=d(v, A)$, and consider separately three cases: (1) $\alpha \leqq \alpha_{0}$ for a suitable small $\alpha_{0}$, (2) $\alpha_{0}<\alpha \leqq 1 / b_{1}^{\prime}$, (3) $\alpha>1 / b_{1}^{\prime}$.

5.17. Theorem. Let $A \subset R^{n}$ be a compact (n-1)-dimensional $C^{1}$-manifold, with or without boundary. Then $A$ has the extension properties in $R^{n}$.

Proof. If $n=1$, then $A$ is a finite set, and the result is obvious. Suppose $n \geqq 2$. For every $y \in A$, let $T(y)$ be the tangent $(n-1)$-plane of $A$ at $y$, and let $P_{y}: R^{n} \rightarrow$ $T(y)$ be the orthogonal projection. For $t>0$, set

$$
D(y, t)=T(y) \cap B^{n}(y, t), \quad Z(y, t)=P_{y}^{-1} D(y, t) .
$$

Let $A(y, t)$ be the $y$-component of $A \cap Z(y, t)$. There it $t_{0}>0$ such that if $t \leqq t_{0}$, then $P_{y} \mid A(y, t)$ is injective and $A \cap B^{n}(y, t) \subset A(y, t)$. By compactness, we can choose $t_{0}$ to be independent of $y$. If $\partial A=\emptyset$, we have $P_{y} A(y, t)=D(y, t)$, but in any case, we can choose $t_{0}$ so that for $t \leqq t_{0}, P_{y} A(y, t)=C(y, t)$ contains a regular $(n-1)$ simplex $\Delta$ with $d(\Delta)=t / 2$.

Let $\varphi_{y}: C(y, t) \rightarrow A(y, t)$ be the local inverse of $P_{y}$, satisfying $P_{y} \varphi_{y}=\mathrm{id}$. By differentiability, we can write

$$
\left|\varphi_{y}(y+h)-(y+h)\right| \leqq|h| \varepsilon(|h|),
$$

where $\varepsilon:\left[0, t_{0}\right] \rightarrow R^{1}$ is an increasing function and $\varepsilon(t) \rightarrow 0$ as $t \rightarrow 0$. By compactness, $\varepsilon$ can be chosen to be independent of $y$.

We show that $A$ satisfies the condition (a) of 5.5 with $b_{1}=3$. Let $0 \leqq \lambda \leqq 1$. Choose $t_{1}, 0<t_{1} \leqq t_{0}$, such that $\varepsilon\left(t_{1}\right) \leqq \lambda / 5$, and sat $r_{0}=t_{1} / 5$. Assume that $x \in R^{n} \backslash A$ with $d(x, A)=r \leqq r_{0}$. Choose $y \in A$ with $|x-y|=r$. Then, with the notation of 5.5 we have

$$
E(x, 3) \subset A \cap B(y, 5 r) \subset A(y, 5 r) \subset T(y)+\lambda r \bar{B}^{n} .
$$

Let $\Delta_{1}$ be a regular $(n-1)$-simplex in $C(y, r)$ with $d\left(\Delta_{1}\right)=r / 2$, and let $\Delta$ be the simplex with $\Delta^{0}=\varphi_{y} \Delta_{1}^{0}$. Since $\varepsilon(r) \leqq 1 / 5$, (5.18) implies that $d(\Delta) \leqq 2 d\left(\Delta_{1}\right)$. Since $b(\Delta) \geqq b\left(\Delta_{1}\right)$, we have

$$
\varrho(\Delta) \leqq 2 \varrho\left(\Delta_{1}\right)=\varrho_{0},
$$

where $\varrho_{0}$ depends only on $n$. Furthermore, for every $z \in A(y, r)$ we have

$$
|z-x| \leqq\left|z-P_{y} z\right|+\left|P_{y} z-y\right|+|y-x| \leqq r \varepsilon(r)+r+r<3 r .
$$


Hence $A(y, r) \subset E(x, 3)$, which implies that $\Delta$ is a simplex of $E(x, 3)$. The theorem follows now from 5.5 .

5.19. Theorem. Let $n \geqq 2$, and let $A \subset R^{n}$ be a finite union of simplexes of dimensions $n$ and $n-1$. Then $A$ has the extension properties in $R^{n}$.

Proof. Suppose that $A$ is a finite union of $n$-simplexes $\sigma_{j}$ and $(n-1)$-simplexes $\Delta_{k}$. We show that the conditions of 5.5 are satisfied with $b_{1}=2, b_{2}=3$.

Let $M_{0}$ be the maximum of all numbers $\varrho\left(\sigma_{j}\right)$ and $\varrho\left(\Delta_{k}\right)$, and let $c_{1}$ and $c_{2}$ be the minimum and the maximum, respectively, of the diameters of the simplexes. Choose $\alpha>0$ such that if $H$ is a component of $R^{n} \backslash T\left(\Delta_{j}\right)$ and if $\Delta_{k}$ meets $H$, then $\Delta_{k}$ has a vertex $v$ in $H$ with $d\left(v, T\left(\Delta_{j}\right)\right) \geqq \alpha$. Set $r_{0}=\min \left(c_{1} / 3, \alpha / 4\right)$.

Let $x \in R^{n} \backslash A$ with $d(x, A)=r \leqq r_{0}$. We divide the proof into three cases:

Case 1. $E(x, 2)$ is contained in some $(n-1)$-plane $T$. Now the condition $\left(\mathrm{a}_{3}\right)$ of 5.5 is trivially true for all $\lambda$. Choose $y \in A$ with $|y-x|=r$. Then $y \in \Delta_{j}$ for some $j$. There is an $(n-1)$-simplex $\Delta$ which is similar to $\Delta_{j}$ and satisfies the conditions

$$
y \in \Delta \subset \Delta_{j} \cap \bar{B}(x, 2 r), d(\Delta) \geqq r .
$$

Hence (a) is true with $M=M_{0}$.

Case 2. $E(x, 2)$ meets $\sigma_{j}$ for some $j$. Now there is an $n$-simplex $\sigma$ which is similar to $\sigma_{j}$ and satisfies the conditions

$$
\sigma \subset \sigma_{j} \cap \bar{B}(x, 3 r), d(\sigma) \geqq r .
$$

Obviously $\sigma$ is $M_{0}$-related to $\sigma_{j}$ in $\sigma_{j}$ and hence in $A$. Thus (b) is true with $M=$ $\max \left(M_{0}, 1 / c_{1}\right)$.

Case 3. The cases 1 and 2 do not occur. Now $E(x, 2)$ meets two $(n-1)$-simplexes $\Delta_{j}$ and $\Delta_{k}$ which are not contained in an $(n-1)$-plane. Choose $y \in A$ with $|x-y|=r$. We may assume that $y \in L_{j}$. To simplify notation, we assume that $y=0$ and that $T\left(\Delta_{j}\right)=R^{n-1}$. Choose $z_{0} \in L_{k} \cap \bar{B}(x, 2 r)$. We may assume that $z_{0} \in R_{+}^{n}$ and that $\Delta_{k} \cap$ int $R_{+}^{n} \neq \emptyset$. Let $P: R^{n} \rightarrow R^{1}$ be the projection $P(x)=x_{n}$, and choose a point $v \in \Lambda_{k}$ at which $P$ attains its maximum. Then $P(v) \geqq \alpha$. Since $\left|v-z_{0}\right| \geqq \alpha-3 r \geqq r$, there is a point $z$ on the segment $v z_{0}$ with $\left|z-z_{0}\right|=r$. Choose $\mu \in(0,1]$ such that the $(n-1)$-simplex $\Delta_{0}=\mu \Delta_{j}$ is contained in $\bar{B}(r)$ and meets $S(r)$. Then $r \leqq d\left(\Delta_{0}\right) \leqq$ $2 r$ and thus $r / c_{2} \leqq \mu \leqq 2 r / c_{1}$. Furthermore, $\sigma=z \Delta_{0}$ is an $n$-simplex of $E(x, 3)$. It suffices to show that $\sigma$ is $M$-related to $v \Delta_{j}$ in $\Delta_{j} \cup \Delta_{k}$ for some $M$ depending only on $A$. This is done by deforming $z \Delta_{0}$ to $v \Delta_{j}$ by a parameter $t \in[0,1]$ so that an intermediate simplex is $\sigma_{t}=z_{t} \Delta_{t}$ where

$$
z_{t}=(1-t) z+t v, \quad \Delta_{t}=\mu_{t} \Delta_{0}, \quad \mu_{t}=1-t+t / \mu .
$$

By 2.15 , it suffices to find an upper bound for the numbers

$$
\beta_{1}=\frac{d\left(z_{t}, \Delta_{t}\right)}{d\left(\bar{\Delta}_{t}\right)}, \quad \beta_{2}=\frac{d\left(\Delta_{t}\right)}{P\left(z_{t}\right)} .
$$


We have

$$
\beta_{1} \leqq \frac{\left|z_{t}\right|}{\mu_{t} r} \leqq \frac{(1-t)|z|+t|v|}{(1-t+t / \mu) r}=\gamma_{1}(t) .
$$

Since $\gamma_{1}$ is monotone and since

$$
\begin{gathered}
\gamma_{1}(0)=\frac{|z|}{r} \leqq \frac{\left|z-z_{0}\right|+\left|z_{0}\right|}{r} \leqq 4, \\
\gamma_{1}(1)=\frac{\mu|v|}{r} \leqq 2\left(\left|v-z_{0}\right|+\left|z_{0}\right|\right) / c_{1} \leqq 4 c_{2} / c_{1},
\end{gathered}
$$

we obtain $\beta_{1} \leqq 4 c_{2} / c_{1}$. To estimate $\beta_{2}$ observe first that

$$
\frac{\left|z-z_{0}\right|}{\left|v-z_{0}\right|}=\frac{P(z)-P\left(z_{0}\right)}{P(v)-P\left(z_{0}\right)} \leqq \frac{P(z)}{P(v)} \leqq \frac{P(z)}{\alpha},
$$

which implies $P(z) \geqq \alpha r / c_{2}$. Since

$$
\beta_{2}=\frac{\mu_{t} d\left(\Delta_{0}\right)}{P\left(z_{t}\right)} \leqq \frac{2 r(1-t+t / \mu)}{(1-t) P(z)+t P(v)},
$$

we can argue as above and obtain $\beta_{2} \leqq 2 c_{2} / \alpha$.

5.20. Corollary. Let $n \geqq 2$, and let $A \subset R^{n}$ be a compact PL manifold of dimension $n$ or $n-1$, with or without boundary. Then $A$ has the extension properties in $R^{n}$.

5.21. Remarks. It is not possible to extend 5.17 and 5.20 to LIP manifolds. For example, a LIP circle in $R^{2}$ need not have the extension properties in $R^{2}$, see 7.10.

One can also consider bilipschitz and QS extension without the condition that the bilipschitz constants or the dilatations are small. For example, Gehring $\left[\mathrm{Ge}_{1}\right.$, Corollary 2, p. 218] proved that if $A$ is a QS circle in $R^{2}$, every $L$-bilipcshitz $f: A \rightarrow R^{2}$ can be extended to an $L_{1}$-bilipschitz $g: R^{2} \rightarrow R^{2}, L_{1}=L_{1}(L, A)$. In higher dimensions $n \neq 4$, similar extension is possible if, for example, $A$ and $f A$ are QC spheres, see $\left[\mathrm{TV}_{5}, 2.19\right]$.

5.22. Open problems. 1. Are 5.17 and 5.20 true for $p$-dimensional manifolds, $p \leqq n-2$ ?

2. Does $A$ in 5.17 and in 5.20 have the extension properties in $\left(R^{n}, Y\right)$ for $\operatorname{dim} Y>n$ ?

3. Does every compact polyhedron in $R^{n}$ have the extension properties in $R^{n}$ ?

5.23. Example. Let $A \subset R^{2}$ be the well-known snow-flake curve see e.g. [Ma, p. 42]. There is a family of equilateral triangles associated with $A$ in a natural way. It is easy to see that these are mutually $M$-related in $A$ with some $M$. It follows from 5.5 that $A$ has the extension properties in $R^{2}$. A stronger result will be given in 6.13.2. 


\section{Thick sets}

6.1. In this section we give a sufficient condition for a set $A \subset R^{p}$ to have the extension properties in $\left(R^{n}, Y\right)$ for $p \leqq n \leqq \operatorname{dim} Y$. The condition is somewhat similar to the condition (b) of Theorem 5.5, but it does not involve the notion of $M$-relatedness. On the other hand, it must be valid at all boundary points and there is no choice between two conditions as in 5.5. We show that the condition applies, for example, to all compact convex sets and to QS $p$-cells.

We say that a set $A \subset R^{p}$ is thick in $R^{p}$ if there are $r_{0}>0$ and $\beta>0$ such that if $y \in \partial A$ and if $0<r \leqq r_{0}$, then there is a $p$-simplex $\Delta$ such that $\Delta^{0} \subset A \cap \bar{B}(y, r)$ and $m_{p}(\Delta) \geqq \beta r^{p}$. This implies that $\varrho(\Delta) \leqq M$ and $d(\Delta) \geqq r / M$ for some $M=M(\beta, p)$. Conversely, these inequalities imply that $m_{p}(\Delta) \geqq \beta r^{p}$ with $\beta=\beta(M, p)>0$. Examples of thick sets are given in 6.13.

6.2. Theorem. Suppose that $A$ is closed and thick in $R^{p}$ and that either $A$ or $R^{p} \backslash A$ is bounded. Then $A$ has the extension properties in $\left(R^{n}, Y\right)$ whenerer $Y$ is a linear subspace of $l_{2}$ and $p \leqq n \leqq \operatorname{dim} Y$.

Proof. By 4.4 it suffices to show that $A$ has the extension properties in $\left(R^{p}, Y\right)$. We again choose an auxiliary parameter $q \in(0,1]$. To prove the QSEP it suffices to find $q_{0} \in(0,1]$ and for every $q \in\left(0, q_{0}\right]$ a number $s=s(q, A)>0$ such that every $s-\mathrm{QS}$ map $f: A \rightarrow Y$ has an $s_{1}-\mathrm{QS}$ extension $g: R^{p} \rightarrow Y$ where $s_{1}=s_{1}(q, A) \rightarrow 0$ as $q \rightarrow 0$. In the bilipschitz case, we find $L=L(q, A)>1$ such that every $L$-bilipschitz $f: A \rightarrow Y$ has an $L_{1}$-bilipschitz extension $g: R^{p} \rightarrow Y$ where $L_{1}=L_{1}(q, A) \rightarrow 1$ as $q \rightarrow 0$.

The basic idea of the proof is the same as in 5.5. However, the number $b$ corresponding to the constant $b_{2}$ of 5.5 will depend on $q$. In fact, $b \rightarrow \infty$ as $q \rightarrow 0$. No use will be made of sense-preservation.

Let $r_{0}>0$ and $\beta>0$ be the numbers given in the definition of thickness, and let $M=M(\beta, p) \geqq 1$ be as in 6.1 . Set

$$
c=q^{-1 / 3}, \quad b=2+3 c,
$$

and choose $R>0$ such that $\partial A \subset \bar{B}^{p}(R / b)$. Choose $s=s(q, A) \in(0, q]$ such that

$$
\varkappa(s, p) \leqq \min \left(q^{2} r_{0} / 2 R, q / 2 b\right),
$$

where $x$ is given by 3.1. We show that $s$ is the required number provided that $q$ is sufficiently small. In the bilipschitz case we set $L=\left(s^{2}+1\right)^{1 / 2}$.

We may assume that $R^{p} \subset Y$. Suppose that $f: A \rightarrow Y$ is $s-\mathrm{QS}$ By 3.1, there is a similarity $h: R^{p} \rightarrow Y$ such that setting $B=\bar{B}^{p}(R)$ we have

$$
\|h-f\|_{A \cap B} \leqq \varkappa(s, p) L_{h} d(A \cap B) \leqq q^{2} r_{0} L_{h} .
$$

Extending $h$ to a bijective similarity $h_{1}$ of $Y$ and replacing $f$ by $h_{1}^{-1} f$ we may assume that

$$
\|f-\mathrm{id}\|_{A \cap B} \leqq q^{2} r_{0} .
$$


Set $G=R^{p} \backslash A$. For every $x \in R^{p}$ we set $r_{x}=d(x, A)$ and choose $a_{x} \in A$ with $\left|a_{x}-x\right|=r_{x}$. We also set $E_{x}=A \cap \bar{B}\left(x, b r_{x}\right)$. Then clearly $E_{x} \subset B$ for all $x \in G$.

We first show that

$$
r_{y} \leqq(1+c) r_{x}, A \cap \bar{B}\left(a_{y}, r_{y}\right) \subset E_{x} \cap E_{y}
$$

whenever $x, y \in G$ with $|y-x| \leqq c r_{x}$. The first inequality is obvious. If $z \in A \cap$ $\bar{B}\left(a_{y}, r_{y}\right)$, then

$$
|z-x| \leqq\left|z-a_{y}\right|+\left|a_{y}-y\right|+|y-x| \leqq r_{y}+r_{y}+c r_{x} \leqq b r_{x} .
$$

Hence $z \in E_{x}$. Furthermore, since $b \geqq 5$, we have

$$
|z-y| \leqq\left|z-a_{y}\right|+\left|a_{y}-y\right| \leqq b r_{y},
$$

which implies $z \in E_{y}$ and proves (6.5).

We associate to every $x \in G$ a similarity $h_{x}: R^{p} \rightarrow Y$ as follows: If $r_{x} \geqq q r_{0}$, we set $h_{x}=$ id. If $r_{x}<q r_{0}$, we apply 3.1 and choose $h_{x}$ such that

$$
\left\|h_{x}-f\right\|_{E_{x}} \leqq \chi(s, p) L\left(h_{x}\right) d\left(E_{x}\right) \leqq 2 b r_{x} L\left(h_{x}\right) \varkappa(s, p) .
$$

By (6.3) this yields

$$
\left\|h_{x}-f\right\|_{E_{x}} \leqq q r_{x} L\left(h_{x}\right)
$$

By (6.4), this is valid for all $x \in G$. In the bilipschitz case $h_{x}$ is chosen to be an isometry.

In what follows, we let $M_{1}, M_{2}, \ldots$ denote numbers $M_{j} \geqq 1$ depending only on $A$. We next show that

$$
L\left(h_{y}\right) r_{y} \leqq 5 M b L\left(h_{x}\right) r_{x},\left|h_{x}(y)-h_{y}(y)\right| \leqq M_{1} q^{2 / 3} r_{x} L\left(h_{x}\right)
$$

whenever $x, y \in G,|y-x| \leqq c r_{x}$ and $r_{x} \leqq r_{0} /(1+c)$.

By (6.5), we have $r_{y} \leqq(1+c) r_{x} \leqq r_{0}$. Hence there is a $p$-simplex $\Delta$ of $A \cap \bar{B}\left(a_{y}, r_{y}\right)$ such that $d(\Delta) \geqq r_{y} / M$ and $\varrho(\Delta) \leqq M$. By (6.5) we have $\Delta^{0} \subset E_{x} \cap E_{y}$. We give the restriction

Now (6.6) implies

$$
q \leqq 1 / 4 M
$$

Hence

$$
\begin{aligned}
L\left(h_{y}\right) r_{y} & \leqq M L\left(h_{y}\right) d(\Delta)=M d\left(h_{y} \Delta\right) \\
& \leqq M\left(d\left(f \Delta^{0}\right)+2 q r_{y} L\left(h_{y}\right)\right) \\
& \leqq M d\left(f \Delta^{0}\right)+r_{y} L\left(h_{y}\right) / 2 .
\end{aligned}
$$

$$
L\left(h_{y}\right) r_{y} \leqq 2 M d\left(f \Delta^{0}\right) \leqq 2 M\left(L\left(h_{x}\right) d(\Delta)+2 q r_{x} L\left(h_{x}\right)\right) .
$$

Since $d(\Delta) \leqq d\left(E_{x}\right) \leqq 2 b r_{x}$ and since $q \leqq 1 \leqq b / 4$, we obtain the first inequality of (6.7). 
To prove the second inequality, we first obtain

Since

$$
\begin{aligned}
\left\|h_{x}-h_{y}\right\|_{\Delta^{0}} & \leqq\left\|h_{x}-f\right\|_{E_{x}}+\left\|f-h_{y}\right\|_{E_{y}} \\
& \leqq q r_{x} L\left(h_{x}\right)+q r_{y} L\left(h_{y}\right) \\
& \leqq(1+5 M b) q r_{x} L\left(h_{x}\right) .
\end{aligned}
$$

$$
1+5 M b \leqq 6 M b \leqq 3 C M c=30 M q^{-1 / 3},
$$

and since $\varrho(\Delta) \leqq M, 2.11$ yields

$$
\left|h_{x}(y)-h_{y}(y)\right| \leqq 30 M q^{2 / 3} r_{x} L\left(h_{x}\right)\left(1+d(\Delta)^{-1} M_{2}\left|y-z_{1}\right|\right),
$$

where $z_{1}$ is an arbitrary vertex of $\Delta$. Since $d(\Delta) \geqq r_{y} / M$ and $\left|y-z_{1}\right| \leqq\left|y-a_{y}\right|+$ $\left|a_{y}-z_{1}\right| \leqq 2 r_{y}$, we obtain the second inequality of (6.7).

Choose a Whitney triangulation $W$ of $G$ as in 5.1. Thus the $p$-simplexes $\sigma$ of $W$ satisfy the conditions (5.2):

$$
\varrho(\sigma) \leqq \varrho_{p}, \quad a_{1} \leqq r_{G}(\sigma) \leqq a_{2},
$$

where $\varrho_{p}, a_{1}, a_{2}$ depend only on $p$. We may assume that $a_{2} \leqq 1$. As in the proof of 5.5 , we define $g(v)=h_{v}(v)$ for every vertex $v$ of $W$, extend affinely to all simplexes of $W$, and set $g \mid A=f$. We shall show that $g$ is the desired extension of $f$ provided that $q$ is sufficiently small.

Since $a_{2} \leqq 1$, we see that $g(x)=x$ whenever $r_{x} \leqq 2 q r_{0}$.

We omit the proof for the continuity of $g$, since it is similar to the corresponding proof in 5.5.

We first show that

$$
\left|h_{x}(x)-g(x)\right| \leqq M_{1} q^{2 / 3} r_{x} L\left(h_{x}\right)
$$

whenever $x \in G$ and $r_{x} \leqq r_{0} /(1+c)$. Choose a $p$-simplex $\sigma \in W$ containing $x$. For every vertex $v$ of $\sigma$ we have

Hence (6.7) implies

$$
|v-x| \leqq d(\sigma) \leqq a_{2} r_{x} \leqq c r_{x} .
$$

$$
\left|h_{x}(v)-g(v)\right| \leqq M_{1} q^{2 / 3} r_{x} L\left(h_{x}\right) .
$$

Since $h_{x}-g$ is affine in $\sigma$, this yields (6.8).

We next show that

$$
\left|h_{x}(y)-g(y)\right| \leqq M_{3} q^{1 / 3} r_{x} L\left(h_{x}\right)
$$

whenever $x \in G, r_{x} \leqq r_{0} /(1+c)^{2}$ and $|y-x| \leqq c r_{x}$. If $y \in A$, then $y \in E_{x}$, and (6.9) follows from (6.6) with $M_{3}=1$. Suppose that $y \in G$. Since $r_{y} \leqq(1+c) r_{x} \leqq r_{0} /(1+c)$, (6.7) and (6.8) imply

$$
\begin{aligned}
\left|h_{x}(y)-g(y)\right| & \leqq\left|h_{x}(y)-h_{y}(y)\right|+\left|h_{y}(y)-g(y)\right| \\
& \leqq M_{1} q^{2 / 3} r_{x} L\left(h_{x}\right)+M_{1} q^{2 / 3} r_{y} L\left(h_{y}\right) .
\end{aligned}
$$

Since $b \leqq 5 c=5 q^{-1 / 3},(6.9)$ follows from the first inequality of (6.7) with $M_{3}=26 M M_{1}$. 
We must show that $g$ is $s_{1}-\mathrm{QS}$, where $s_{1}=s_{1}(q, A) \rightarrow 0$ as $q \rightarrow 0$. In the bilipschitz case, we must show that $g$ is $L_{1}$-bilipschitz, where $L_{1}=L_{1}(q, A) \rightarrow 1$ as $q \rightarrow 0$. We omit the proof of the QS case, since it would take several pages of elementary and dull reasoning, where one would consider several cases and subcases according to the situation of a triple $(a, b, x)$ of points in $R^{p}$. We give in detail the proof for the bilipschitz case, which is simpler. Assume that $f: A \rightarrow Y$ is $L$-bilipschitz satisfying (6.3) with $s=\left(L^{2}-1\right)^{1 / 2}$. Now each $h_{x}$ is an isometry, and thus $L\left(h_{x}\right)=1$.

For every $p$-simplex $\sigma$ of $W$, we let $K_{\sigma}$ denote the subcomplex of $W$ generated by all $p$-simplexes meeting $\sigma$. The underlying polyhedron $N(\sigma)=\left|K_{\sigma}\right|$ is a neighborhood of $\sigma$ in $R^{p}$. From the construction of $W$ it follows that there are positive numbers $a_{3}$ and $a_{4}$ depending only on $p$ such that

$$
d\left(\sigma, R^{p} \backslash N(\sigma)\right) \geqq a_{3} r_{x}, \quad|y-x| \leqq a_{4} r_{x},
$$

whenever $\sigma \in W$ is a $p$-simplex, $x \in \sigma$, and $y \in N(\sigma)$. Moreover, the complexes $K_{\sigma}$ belong to a finite number of similarity classes. By 2.14 , there exist a number $\alpha_{0}=$ $\alpha_{0}(p)>0$ and for every $\alpha \in\left(0, \alpha_{0}\right]$ a number $L_{2}=L_{2}(\alpha, p)$ such that $L_{2}(\alpha, p) \rightarrow 1$ as $\alpha \rightarrow 0$ and such that if $\varphi: N(\sigma) \rightarrow l_{2}$ is affine on each simplex of $K(\sigma)$ and if $\mid \varphi(v)-$ $h(v) \mid \leqq \alpha d(\sigma)$ for some isometry $h: R^{p} \rightarrow l_{2}$ and for every vertex $v$ of $K(\sigma)$, then $\varphi$ is $L_{2}$-bilipschitz.

We give the following new restrictions on $q$ :

$$
q \leqq a_{4}^{-3}, \quad q \leqq \alpha_{0}^{3} a_{1}^{3} M_{3}^{-3}, \quad 2 q \leqq(1+c)^{-2},
$$

which are of the form $q \leqq q_{0}(A)$. We show that for every $p$-simplex $\sigma \in W, g \mid N(\sigma)$ is $L_{3}$-bilipschitz with $L_{3}=L_{3}(q, A)=L_{2}\left(M_{3} q^{1 / 3} / a_{1}, p\right)$. Choose $x \in \sigma$ with $r_{x}=$ $d(\sigma, A)$. If $r_{x} \geqq r_{0} /(1+c)^{2}$, the last inequality of (6.10) implies that $r_{y} \geqq q r_{0}$ for all $y \in N(\sigma)$, and hence $g \mid N(\sigma)=$ id. If $r_{x} \leqq r_{0} /(1+c)^{2}$, then (6.10) implies that $N(\sigma) \subset$ $B\left(x, c r_{x}\right)$. Hence $(6.9)$ yields

$$
\left|h_{x}(y)-g(y)\right| \leqq \alpha d(\sigma)
$$

for $y \in N(\sigma)$ with $\alpha=M_{3} q^{1 / 3} / a_{1} \leqq \alpha_{0}$. Thus $g \mid N(\sigma)$ is $L_{3}$-bilipschitz.

It follows that $g$ is $L_{4}$-lipschitz with $L_{4}=L_{4}(q, A)=\max \left(L, L_{3}\right)$. We assume that $q$ is so small that $L_{4} \leqq 2$. It remains to find $L_{5}=L_{5}(q, A)$ such that

$$
|g(x)-g(y)| \geqq|x-y| / L_{5}
$$

for all $x, y \in R^{p}$ and such that $L_{5}(q, A) \rightarrow 1$ as $q \rightarrow 0$. We may assume that $r_{y} \leqq r_{x}$. We consider three cases.

Case 1. $r_{x}=0$. Now $x, y \in A$, and (6.11) holds with $L_{5}=L$.

Case 2. $0<r_{x} \leqq r_{0} /(1+c)^{2}$. Choose a $p$-simplex $\sigma \in W$ containing $x$. If $y \in N(\sigma)$, (6.11) holds with $L_{5}=L_{3}$. If $y \in B\left(x, c r_{x}\right) \backslash N(\sigma)$, then $|x-y| \geqq a_{3} r_{x}$, and (6.9) 
yields

$$
\begin{aligned}
|g(x)-g(y)| & \geqq\left|h_{x}(x)-h_{x}(y)\right|-\left|h_{x}(x)-g(x)\right|-\left|h_{x}(y)-g(y)\right| \\
& \geqq|x-y|-2 M_{3} q^{1 / 3} r_{x} \\
& \geqq|x-y|\left(1-2 M_{3} q^{1 / 3} / a_{3}\right),
\end{aligned}
$$

which implies (6.11) for small $q$. Finally assume that $|x-y| \geqq c r_{x}$. Since $g$ is 2-lipschitz, we obtain

$$
\begin{aligned}
|g(x)-g(y)| & \geqq\left|g\left(a_{x}\right)-g\left(a_{y}\right)\right|-\left|g(x)-g\left(a_{x}\right)\right|-\left|g(y)-g\left(a_{y}\right)\right| \\
& \geqq\left|a_{x}-a_{y}\right| / L-2 r_{x}-2 r_{y} \\
& \geqq\left(|x-y|-2 r_{x}\right) / L-4 r_{x} \\
& \geqq|x-y|\left(1-2 q^{1 / 3}\right) / L-4 q^{1 / 3}|x-y|,
\end{aligned}
$$

which gives (6.11) for small $q$.

Case 3. $r_{x} \geqq r_{0} /(1+c)^{2}$. Now $g(x)=x$. If $r_{y} \geqq 2 q r_{0}$, then $g(y)=y$, and (6.11) is trivial. Assume that $r_{y} \leqq 2 q r_{0}$. If $y \in G$ or if $|y| \leqq R$, (6.4) gives

$$
\begin{aligned}
|g(x)-g(y)| & \geqq|x-y|-\left|y-a_{y}\right|-\left|a_{y}-g\left(a_{y}\right)\right|-\left|g\left(a_{y}\right)-g(y)\right| \\
& \geqq|x-y|-2 q r_{0}-q^{2} r_{0}-2\left|a_{y}-y\right| \\
& \geqq|x-y|-7 q r_{0} .
\end{aligned}
$$

Since $|x-y| \geqq r_{0} /(1+c)^{2}-2 q r_{0}$, we again obtain (6.11) for small $q$. Finally, assume that $y \in A$ and $|y| \geqq R$. Now $r_{x} \leqq R / b$ and $|x-y| \geqq R-R / b$. Since $g$ is 2-lipschitz and since $b-1=1+3 c>q^{1 / 3}$, we obtain

$$
\begin{aligned}
|g(x)-g(y)| & \geqq\left|g\left(a_{x}\right)-g(y)\right|-\left|g(x)-g\left(a_{x}\right)\right| \\
& \geqq\left|a_{x}-y\right| / L-2 r_{x} \\
& \geqq|x-y|\left(1-q^{1 / 3}\right) / L-2 q^{1 / 3}|x-y|,
\end{aligned}
$$

which again implies (6.11) for small $q$.

6.12. Remarks. Inspection of the proof of 6.2 gives the following information on the constants $L_{0}$ and $L_{1}$ of the BLEP: $L_{0}$ depends only on $r_{0} / d(\partial A), \beta$ and $n$, and $L_{1}$ depends, in addition, only on $L$. In particular, these numbers do not depend on $Y$. In the case $p=n$, we can choose $g$ to be an isometry outside a given neighborhood $U$ of $A$; then $L_{0}$ depends also on $U$.

A similar statement is true for the QSEP. Then one can choose $g \mid R^{\nwarrow \backslash U}$ to be a similarity.

6.13. Examples. 1. Suppose that a domain $D \subset R^{p}$ is a John domain, see e.g. [MS]. It is then easy to see that $\bar{D}$ is thick in $R^{p}$, and has therefore the extension properties in $\left(R^{n}, Y\right)$. In particular, this is true if $D$ is a bounded uniform domain [GM, 2.18]; in particular, if $D$ is a QS ball [V $\left.\ddot{a}_{2}, 5.6\right]$; in particular if $D$ is bounded 
and convex. It follows that every convex compact set in $R^{n}$ has the extension properties in $\left(R^{n}, Y\right)$.

2. Let $A$ be the snow-flake curve in $R^{2}$. It is easy to see that $A$ is thick in $R^{2}$, and has thus the extension properties in $\left(R^{n}, Y\right)$ for $2 \leqq n \leqq \operatorname{dim} Y$. This strengthens the result of 5.23. Since $A$ is a QS circle, we see that thickness is not a QS invariant property.

3. The Cantor middle-third set is thick in $R^{1}$.

4. If $A_{1}$ is thick in $R^{p}$ and $A_{2}$ is thick in $R^{q}$, then $A_{1} \times A_{2}$ is thick in $R^{p+q}$.

5. If $A$ is any closed set in $R^{p}$ and if $r_{0}>0$, then $A+r_{0} \bar{B}^{p}$ is thick in $R^{p}$ with constants $r_{0}$ and $\beta=\beta(p)$. This observation will be used in Section 8 .

\section{Examples}

7.1. In this section we give several examples of sets $A \subset R^{n}$ which have neither of the extension properties in $R^{n}$ or in $\left(R^{n}, Y\right)$ for some $Y$. To show this, it suffices to construct a sequence of $L_{k}$-bilipschitz maps $f_{k}: A \rightarrow Y$ such that $L_{k} \rightarrow 1$ and such that there are no $s_{k}-\mathrm{QS}$ extensions $g_{k}: R^{n} \rightarrow Y$ of $f_{k}$ such that $s_{k} \rightarrow 0$. In 7.5, we give an example of a set which has the BLEP but not the QSEP in $R^{2}$.

7.2. Lemma. Let $n \geqq 2$, let $1 \leqq L<b$, and let $x_{0}, y_{0}$ be points in $R^{n} \backslash\{0\}$ such that $\left|x_{0}\right| / L \leqq\left|y_{0}\right| \leqq L\left|x_{0}\right|$. Then there is an $L_{1}$-bilipschitz maph: $R^{n} \rightarrow R^{n}$ such that

(1) $h\left(x_{0}\right)=y_{0}$,

(2) $h(x)=x$ if $|x| \leqq\left|x_{0}\right| / b$ or $|x| \geqq b\left|x_{0}\right|$,

(3) $L_{1}=L_{1}(L, b) \rightarrow 1$ as $L \rightarrow 1$ and $b \rightarrow \infty$.

If, in addition, $\left|y_{0}-x_{0}\right| \leqq \delta\left|x_{0}\right|$, one can replace (3) by

(3') $L_{1}=L_{1}(\delta, b) \rightarrow 1$ as $\delta \rightarrow 0$.

Proof. We may assume $n=2$. The map $h$ can be constructed as the map $f$ on $\mathrm{p}$. 205 of $\left[\mathrm{Ge}_{1}\right]$, combined with a simple radial map. The last statement is clear.

7.3. Let $x_{1}, x_{2}, \ldots$ be a strictly decreasing sequence of positive numbers such that $x_{k+1} / x_{k} \rightarrow 0$ and thus $x_{k} \rightarrow 0$. Then $A=\{0\} \cup\left\{x_{k}: k \in N\right\}$ has neither of the extension properties in $R^{1}$. To see this, define $f_{k}: A \rightarrow R^{1}$ by $f_{k}\left(x_{k}\right)=-x_{k}$ and by $f_{k}(x)=x$ for $x \neq x_{k}$. Then $f_{k}$ is $L_{k}$-bilipschitz with $L_{k} \rightarrow 1$, but $f_{k}$ has no extension to a homeomorphism $g: R^{1} \rightarrow R^{1}$.

7.4. Let $A$ be as in 7.3. We show that $A$ has the BLEP in $R^{n}$ for $n \geqq 2$. Suppose that $f: A \rightarrow R^{n}$ is $L$-bilipschitz with $L$ close to one. We may assume that $f(0)=0$ and that $\|f-\mathrm{id}\|_{A}$ is small (Theorem 3.1). Choose disjoint annuli $A_{j}=\left\{x \in R^{n}: x_{j} / b_{j}<\right.$ $\left.|x|<b_{j} x_{j}\right\}$ where $b_{j} \rightarrow \infty$ as $j \rightarrow \infty$. Then Lemma 7.2 gives easily an $L_{1}$-bilipschitz extension $g: R^{n} \rightarrow R^{n}$ of $f$ such that $L_{1}$ is close to one. 
7.5. Let $A$ be as in 7.3 and in 7.4 with $x_{n}=e^{-n !}$. We show that $A$ does not have the QSEP in any connected set. In particular, $A$ has the BLEP but not the QSEP in $R^{2}$. Fix a positive integer $k$, and define a map $f_{k}: A \rightarrow R^{1}$ as follows: Set $\varphi(x)=$ $-1 / \log x$. Then $f_{k}(0)=0, f_{k}(x)=\varphi(x)$ for $x \leqq x_{k}$, and $f_{k}(x)=\varphi(x)+\varphi^{\prime}\left(x_{k}\right)\left(x-x_{k}\right)$ for $x \geqq x_{k}$. An elementary but tedious proof shows that $f_{k}$ is $s_{k}-$ QS where $s_{k} \rightarrow 0$ as $k \rightarrow \infty$. However, $f_{k}$ has no QS extension to any connected set, since by [ $\left.\mathrm{IV}_{1}, 3.14\right]$. this extension would be Hölder continuous at the origin.

7.6. Let $A \subset R^{2}$ be the union of $R^{1}$ and the line segments $J_{k}=2^{k} \times[0,1], k \in N$. Define $f_{k}: A \rightarrow R^{2}$ by $f_{k}(x, y)=(x,-y)$ for $(x, y) \in J_{k}$ and by $f_{k} \mid\left(A \backslash J_{k}\right)=$ id. Then $f_{k}$ is $L_{k}$-bilipschitz where $L_{k} \rightarrow 1$ as $k \rightarrow \infty$. Since $f_{k}$ has no extension to a homeomorphism of $R^{2}, A$ has neither of the extension properties in $R^{2}$.

7.7. The preceding example can easily be modified to a compact set $A \subset R^{2}$ with the same property. This set consists of the horizontal segment $I=[0,1]$ and of the vertical segments $\{1 / k\} \times\left[0,2^{-k}\right]$.

7.8. We modify the preceding example so that $A$ will be an arc. Set

$$
E=\left\{(x, y) \in R^{2}:|x| \leqq 1, y=1-|x|^{1 / 2}\right\} .
$$

The intervals $\Delta_{k}=\left[1 / k-2^{-k}, 1 / k+2^{-k}\right]$ are disjoint for $k \geqq 7$. Let $A$ be the arc obtained from $I$ by replacing each $\Delta_{k}, k \geqq 7$, by $E_{k}=2^{-k} A+1 / k$. Define again $f_{k}: A \rightarrow R^{2}$ by $f_{k}(x, y)=(x,-y)$ for $(x, y) \in E_{k}$ and by $f_{k} \mid\left(A \backslash E_{k}\right)=$ id. Then $f_{k}$ is $L_{k}$-bilipschitz with $L_{k} \rightarrow 1$. Now $f_{k}$ has an extension to a homeomorphism $g: R^{2} \rightarrow R^{2}$, but $g$ cannot be QC and hence not bilipschitz.

A related example has recently been given by Gehring $\left[\mathrm{Ge}_{2}\right]$.

7.9. We replace the $\operatorname{arc} E$ of 7.8 by the PL arc $E^{\prime}$ with consecutive vertices $-e_{1},-e_{1}+e_{2}, e_{1}+e_{2}, e_{1}$. We obtain an arc $A^{\prime} \subset R^{2}$. Define $f_{k}: A^{\prime} \rightarrow R^{2}$ as before. Again $f_{k}$ is $L_{k}$-bilipschitz with $L_{k} \rightarrow 1$. Now $f_{k}$ has an extension to a bilipschitz homeomorphism $g_{k}: R^{2} \rightarrow R^{2}$, but $g_{k}$ cannot be chosen to be $s_{k}-$ QS with $s_{k} \rightarrow 0$, since $g_{k}$ maps an angle $\pi / 2$ onto an angle $3 \pi / 2$.

Observe that $A^{\prime}$ is a LIP arc, that is, a bilipschitz image of $I$. By 5.17 and 5.10, all DIFF and PL arcs in $R^{2}$ have the extension properties.

7.10. It is easy to enlarge the arc $A^{\prime}$ of the preceding example to a LIP circle $A^{\prime \prime}$ which has neither of the extension properties in $R^{2}$. On the other hand, if $D$ is the bounded component of $R^{2} \backslash A^{\prime \prime}$, then $D$ is a bilipschitz disc, and hence $\bar{D}$ has the extension properties in $R^{2}$ by 6.13.1.

7.11. Similar examples can be given in higher dimensions. For example, a LIP $\operatorname{arc~in} R^{3}$ without the extension properties can be obtained from the preceding example by replacing the arc $E^{\prime}$ by the PL arc with vertices $-e_{1},-e_{1}+e_{2},-e_{1}+e_{2}+e_{3}$, $e_{1}+e_{2}+e_{3}, e_{1}+e_{2}, e_{1}$. 
7.12. We next give an example of a set $A \subset R^{3}$ without the extension properties such that $A$ is the closure of a domain. I do not know whether such an example exists in $R^{2}$. Set

$$
\begin{aligned}
D_{1} & =R^{2} \times(1, \infty), \\
D_{2} & =R^{2} \times(-\infty, 0), \\
Z_{k} & =B^{2}\left(k e_{1}, 1 / k\right) \times I, \\
G & =D_{1} \cup D_{2} \cup Z_{2} \cup Z_{3} \cup \ldots .
\end{aligned}
$$

Now $G$ is a domain in $R^{3}$. We shall prove that $A=\bar{G}$ has neither of the extension properties in $R^{3}$.

For $k \geqq 2$ define a homeomorphism $f_{k}: A \rightarrow A$ as follows: Outside $Z_{k}, f_{k}$ is the identity map. In $Z_{k}, f_{k}$ is the twist

$$
f_{k}\left(k+r e^{i \varphi}, t\right)=\left(k+r e^{i(\varphi+2 \pi t)}, t\right) .
$$

Since the cylinders $Z_{k}$ become very thin for large $k$, it is easy to see that $f_{k}$ is $L_{k}$-bilipschitz with $L_{k} \rightarrow 1$.

We show that $f_{k}$ has no extension to a homeomorphism $g: R^{3} \rightarrow R^{3}$. Suppose that $g$ is such an extension, and assume $k \geqq 3$. Define a path $\alpha: I \rightarrow R^{3}$ by $\alpha(s)=$ $2 e_{1}+s e_{3}$. Next choose a natural path homotopy $H_{t}: I \rightarrow R^{3}$ of $\alpha$ such that $H_{t}$ is a $\mathrm{PL}$ path with vertices $2 e_{1}, x_{t}, x_{t}+e_{3}, 2 e_{1}+e_{3}$, where

$$
x_{t}=(2(1-t)+t(k-1 / k)) e_{1} .
$$

Let $P: R^{3} \rightarrow R^{2}$ be the orthogonal projection. Now $P g H_{t}: I \rightarrow R^{2}$ is a path homotopy in $R^{2} \backslash\left\{k e_{1}\right\}$. Hence $P g H_{1}$ is null-homotopic in $R^{2} \backslash\left\{k e_{1}\right\}$, which is clearly a contradiction.

7.13. We can easily modify the preceding example to a compact set $A$ which consists of a closed 3-ball $B$ together with a sequence of handles $Z_{k}$ which are very thin for large $k$. We can choose these handles so that $d\left(Z_{k}\right) \rightarrow 0$. Now remove a thin slice $E_{k}$ from each handle $Z_{k}$. In the situation of $7.12 E_{k}$ could correspond to the set $B^{2}\left(k e_{1}, 1 / k\right) \times\left(0,2^{-k}\right)$. We obtain a set $Q$, which is a locally flat TOP 3-cell. If $f_{k}: Q \rightarrow Q$ is defined as in 7.12, $f_{k}$ can be extended to a homeomorphism $g: R^{3} \rightarrow R^{3}$. However, one can show that $g$ cannot be QC. Hence $Q$ has neither of the extension properties in $R^{3}$. Remember that by 6.13.1, every QS $n$-cell has the extension properties in $R^{n}$.

7.14. We give an example of a set $A \subset R^{2}$ which has the extension properties in $R^{2}$ but not in $\left(R^{2}, R^{3}\right)$, or equivalently, in $R^{3}$. Let $Q_{0}$ be the square $I \times I$, and set inductively $Q_{k}=\left[a_{k}, a_{k}+1\right] \times I$ where $a_{0}=0, a_{k}=a_{k-1}+1+1 / k$. Removing the squares $Q_{0}, Q_{1}, \ldots$ from $R^{2}$ we obtain a domain $G$. We show that $A=\bar{G}$ has the BLEP in $R^{2}$; the QSEP can be proved in a similar manner. Suppose that $f: A \rightarrow R^{2}$ is $L$-bilipschitz with $L$ close to one. By $5.19, \partial Q_{j}$ has the BLEP in $R^{2}$. Hence $f \mid \partial Q_{j}$ can be extended to an $L_{1}$-bilipschitz $g_{j}: Q_{j} \rightarrow R^{2}$ where $L_{1}=L_{1}(L) \rightarrow 1$ as $L \rightarrow 1$. 
Since each $Q_{j}$ is a square, $L_{1}$ does not depend on $j$. These maps give an extension $g: R^{2} \rightarrow R^{2}$ of $f$, which is $L_{2}$-bilipschitz with $L_{2}=\max \left(L, L_{1}\right)$.

To show that $A$ does not have the extension properties in $\left(R^{2}, R^{3}\right)$ define $f_{k}: A \rightarrow$ $R^{3}$ as follows. Let $R_{k}$ be the rectangle $\left[a_{k}+1, a_{k+1}\right] \times I$. Let $f_{k}$ be the identity outside $R_{k}$, and let $f_{k} \mid R_{k}$ be a twist, see 7.12. Then $f_{k}$ is $L_{k}$-bilipschitz with $L_{k} \rightarrow 1$. As in 7.12, one can show that $f_{k}$ has no extension to a homeomorphism of $R^{3}$.

7.15. Suppose that $X$ and $Y$ are linear subspaces of $l_{2}$ with $\infty>\operatorname{dim} X \leqq \operatorname{dim} Y$, and let $A \subset X$. It is natural to ask how the extension properties of $A$ in $(X, Y)$ depend on $X$ and $Y$. By 4.4, they are independent of $X$. However, the examples 7.3, 7.4 and 7.14 show that they depend essentially on $Y$. More precisely, if $Y_{1} \subset Y_{2}$ with $\operatorname{dim} Y_{1}<$ $\operatorname{dim} Y_{2}$, the extension properties of $A$ in $\left(X, Y_{1}\right)$ do not imply and are not implied by the extension properties of $A$ in $\left(X, Y_{2}\right)$.

7.16. Suppose that $A$ is an infinite-dimensional linear subspace of $l_{2}$ with $\bar{A} \neq l_{2}$. Then there is an isometry $f: A \rightarrow l_{2}$ such that $f A$ is dense in $l_{2}$. Hence $A$ has neither of the extension properties in $l_{2}$. It seems to the author that the notions BLEP and QSEP are only useful for finite-dimensional sets $A$.

\section{Supplementary results}

In this section we give some general remarks on the extension properties. We first show that if $A$ is compact, the extensions can be chosen to be very elementary outside a given neighborhood of $A$.

8.1. Theorem. Suppose that $A \subset R^{n}$ is compact and has the BLEP in $\left(R^{n}, Y\right)$, where $Y$ is a linear subspace of $l_{2}$. Let $U$ be a neighborhood of $A$. Then there is $L_{0}>1$ such that if $1 \leqq L \leqq L_{0}$, then every L-bilipschitz $f: A \rightarrow Y$ has an $L_{1}$-bilipschitz extension $g: R^{n} \rightarrow Y$ such that $L_{1}=L_{1}(L, A, U, n, Y) \rightarrow 1$ as $L \rightarrow 1$ and such that $g \mid R^{n} \backslash U$ is an isometry.

A similar statement is true for the QSEP; then $g \mid R^{n} \backslash U$ is a similarity.

Proof. We prove the first part of the theorem; the proof for the QS case is similar. Let $L_{0}^{\prime}>1$ and $L_{1}^{\prime}(L, A, n, Y)$ be the numbers given by the BLEP of $A$ in $\left(R^{n}, Y\right)$. Set $r_{0}=d(A, \partial U) / 2$ and $E=A+r_{0} \bar{B}^{n}$. By 6.2 and 6.13.5, $E$ has the BLEP in $\left(R^{n}, Y\right)$. More precisely, it follows from 6.12 that there is $L_{0}^{\prime \prime}=L_{0}^{\prime \prime}\left(r_{0} / d(E), n\right)>1$ such that if $1 \leqq L \leqq L_{0}^{\prime \prime}$, then every $L$-bilipschitz $f: E \rightarrow Y$ has an $L_{1}^{\prime \prime}$-bilipschitz extension $g: R^{n} \rightarrow$ $Y$ such that $L_{1}^{\prime \prime}=L_{1}^{\prime \prime}\left(L, r_{0} / d(E), n\right) \rightarrow 1$ as $L \rightarrow 1$ and such that $g \mid R^{n} \backslash U$ is an isometry. Choose $L_{0}>1$ such that $L_{0} \leqq L_{0}^{\prime}$ and such that $L_{1}^{\prime}(L, A, n, y) \leqq L_{0}^{\prime \prime}$ for $1 \leqq L \leqq L_{0}$. Suppose that $1 \leqq L \leqq L_{0}$ and that $f: A \rightarrow Y$ is $L$-bilipschitz. Then $f$ has an $L_{1}^{\prime}(L, A, n, Y)$-bilipschitz extension $h: R^{n} \rightarrow Y$. Now there is an extension $g: R^{n} \rightarrow Y$ of $h \mid E$ such that $g \mid R^{n} \backslash U$ is an isometry and $g$ is $L_{1}$-bilipschitz with

$$
L_{1}=L_{1}^{\prime \prime}\left(L_{1}^{\prime}(L, A, n, Y), r_{0} / d(E), n\right) \text {. }
$$


Observe that the proof of 8.1 made use only of the fact that $A$ has the BLEP in $(U, Y)$. Hence we obtain:

8.2. Theorem. Suppose that $A \subset R^{n}$ is compact, that $U$ is a neighborhood of $A$, that $Y$ is a linear subspace of $l_{2}$, and that $A$ has the BLEP or the QSEP in $(U, Y)$. Then $A$ has the same property in $\left(R^{n}, Y\right)$.

8.3. Remark. On the other hand, there seems to be genuine problems if we consider local extension properties. Suppose, for example, that $A$ is compact in $R^{n}$ and that $Y$ is a linear subspace of $l_{2}$. Suppose also that each point in $A$ has a neighborhood $U$ such that $A \cap \bar{U}$ has the BLEP in $\left(R^{n}, Y\right)$. I do not know whether this implies that $A$ has the BLEP in $\left(R^{n}, Y\right)$.

8.4. Addendum. In a recent paper [Tr], D. A. Trotsenko announces results related to our results on the QSEP. He uses the notion of $h$-similarity, which for small $h$ is close to $s$-quasisymmetry with small $s$, cf. 3.9. However, the examples of Section 7 (e.g. 7.6) seem to contradict Theorem 1 of [ $\mathrm{Tr}]$, unless [ $\mathrm{Tr}$ ] tacitly assumes that all similarities are sense-preserving.

\section{References}

[Do] DoLD, A.: Lectures on algebraic topology. - Springer-Verlag, Berlin-Heidelberg-New York, 1972.

[Ge ${ }_{1}$ Gehring, F. W.: Injectivity of local quasi-isometries. - Comment. Math. Helv. 57, 1982, $202-220$.

[Ge $\left.{ }_{2}\right]$ Gehring, F. W.: Extensions of quasiisometric embeddings of plane arcs and curves. - To appear.

[GM] Gehring, F. W., and O. Martio: Lipschitz classes and quasiconformal mappings. - Ann. Acad. Sci. Fenn. Ser. A I Math. 10, 1985, 203-219.

[Ma] Mandelbrot, B. B.: The fractal geometry of nature. - W. H. Freeman and Company, San Francisco, 1982.

[MS] Martio, O., and J. SARvas: Injectivity theorems in plane and space. - Ann. Acad. Sci. Fenn. Ser. A I Math. 4, 1979, 383-401.

[RR] RADO, T., and P. V. REICHELDERFER: Continuous transformations in analysis. - Die Grundlehren der mathematischen Wissenschaften 75, Springer-Verlag, Berlin-GöttingenHeidelberg, 1955.

[St] Stein, E. M.: Singular integrals and differentiability properties of functions. - Princeton University Press, Princeton, New Jersey, 1970.

[TY] Trus, C. J., and G. S. Young: The extension of interiority, with some applications. - Trans. Amer. Math. Soc. 103, 1962, 329-340.

[Tr] Trotsenko, D. A.: Continuation from a domain and the approximation of space quasiconformal mappings with small distortion coefficient. - Soviet Math. Dokl. 27, 1983, 777780.

[TV $\left.{ }_{1}\right]$ TUkIA, P., and J. VäIsÄLÄ: Quasisymmetric embeddings of metric spaces. - Ann. Acad. Sci. Fenn. Ser. A I Math. 5, 1980, 97-114.

[ $\left.\mathrm{TV}_{2}\right]$ TUKIA, P., and J. VÄISÄLÄ: Lipschitz and quasiconformal approximation and extension. - Ann. Acad. Sci. Fenn. Ser. A I Math. 6, 1981, 303-342. 
[TV ${ }_{3}$ ] TukIA, P., and J. VÄISÄLÄ: Quasiconformal extensicn from dimension $n$ to $n+1$. - Ann. of Math. (2) 115, 1982, 331-348.

[TV $\left.{ }_{4}\right]$ TUKIA, P., and J. VÄISÄLÄ: Extension of $\epsilon$ mbeddings close to iscmetries or similarities. - Ann. Acad. Sci. Fenn. Ser. A I Math. 9, 1984, 153-175.

[TV $\left.{ }_{5}\right]$ TUKIA, P., and J. VÄISÄLÄ: Bilipschitz extensions of maps having quasiconformal extensions. Math. Ann. 269, 1984, 561-572.

[Vä] VÄrsÄLÄ, J.: Lectures on $n$-dimensional quasiconformal mappings. - Lecture Notes in Mathematics 229. Springer-Verlag, Berlin-Heidelterg-N€w York, 1971.

[Vä̈] VÄıs̈̈L̈̈, J.: Quasimöbius maps. - J. Analyse Math. 44, 1984/85, 218-234.

University of Helsinki

Department of Mathematics

SF-00100 Helsinki

Finland

Received 27 March 1985 